\title{
CPT Groups of Spinor Fields in de Sitter and Anti-de Sitter Spaces
}

\author{
V. V. Varlamov*
}

\begin{abstract}
CPT groups for spinor fields in de Sitter and anti-de Sitter spaces are defined in the framework of automorphism groups of Clifford algebras. It is shown that de Sitter spaces with mutually opposite signatures correspond to Clifford algebras with different algebraic structure that induces an essential difference of $C P T$ groups associated with these spaces. CPT groups for charged particles are considered with respect to phase factors on the various spinor spaces related with real subalgebras of the simple Clifford algebra over the complex field (Dirac algebra). It is shown that $C P T$ groups for neutral particles which admit particle-antiparticle interchange and $C P T$ groups for truly neutral particles are described within semisimple Clifford algebras with quaternionic and real division rings, respectively. A difference between bosonic and fermionic $C P T$ groups is discussed.
\end{abstract}

\section{Introduction}

As is known, de Sitter and anti-de Sitter spaces of different dimensions have been extensively studied during the past two decades mainly with the purpose of understanding the correspondence between supergravity in a five-dimensional anti-de Sitter space and $N=4$ supersymmetric field theory in four dimensions. On the other hand, quantum field theory on the de Sitter spacetime by itself at present time is a rapid developing area in theoretical and mathematical physics [1]-[7]. One of the first problem, which naturally arising in this context, is an investigation of CPT groups for spinor fields in de Sitter $\mathbb{R}^{1,4}$ and anti-de Sitter $\mathbb{R}^{3,2}$ spaces. Importance of discrete transformations is well-known, many textbooks on quantum theory began with description of the discrete symmetries, and famous Lüders-Pauli CPT Theorem is a keystone in general structure of quantum field theory (see, for example, the excellent book [8]). Besides, a fundamental notion of antimatter immediately relates with the charge conjugation $C$. However, usual practice of definition of the discrete symmetries from the analysis of relativistic wave equations does not give a full and consistent theory of the discrete transformations. In the standard approach, except a well studied case of the spin $j=1 / 2$ (Dirac equation), a situation with the discrete symmetries

${ }^{*}$ Siberian State Industrial University, Kirova 42, Novokuznetsk 654007, Russia 
in quantum field theory remains unclear for the fields of higher spin $j>1 / 2$. It is obvious that the main reason of this is an absence of a fully adequate formalism for description of higher-spin fields (all widely accepted higher-spin formalisms such as Rarita-Schwinger approach [9], Bargmann-Wigner [10] and Gel'fand-Yaglom [1] multispinor theories, and also Joos-Weinberg $2(2 j+1)$-component formalism [12, 13] have many intrinsic contradictions and difficulties). Moreover, Lee and Wick [14] claimed that "the situation is clearly an unsatisfactory one from a fundamental point of view". The first attempt of going out from this situation was initiated by Gel'fand, Minlos and Shapiro in 1958 [15]. In the Gel'fand-Minlos-Shapiro approach the discrete symmetries are represented by outer involutory automorphisms of the Lorentz group (there are also other realizations of the discrete symmetries via the outer automorphisms, see [16, 17, 18]). At present, the Gel'fand-Minlos-Shapiro ideas have been found further development in the works of Buchbinder, Gitman and Shelepin [19, 20], where the discrete symmetries are represented by both outer and inner automorphisms of the Poincaré group. An algebraic method for description of discrete symmetries was proposed by author in the works [21, 22], where the discrete symmetries are represented by automorphisms of the Clifford algebras.

In this paper $C P T$ groups are considered in the spaces $\mathbb{R}^{4,1}$ and $\mathbb{R}^{1,4}$ with mutually opposite signatures $(+,+,+,+,-)$ and $(-,-,-,-,+)$. The difference of signatures induces difference of Clifford algebras associated with these spaces. So, for the algebra $C \ell_{4,1}$, associated with the space $\mathbb{R}^{4,1}$, there is an isomorphism $C \ell_{4,1} \simeq C \ell_{4}$, where $C \ell_{4}$ is a Dirac algebra, and for the algebra $C \ell_{1,4}$, associated with the space $\mathbb{R}^{1,4}$, we have a decomposition $C \ell_{1,4} \simeq C \ell_{4,0} \oplus C \ell_{4,0}$, where $C \ell_{4,0} \simeq C \ell_{1,4}^{+}$is an even subalgebra of $C \ell_{1,4}$. $C P T$ groups of the algebra $C \ell_{4,1} \simeq C \ell_{4}$ are studied in the section 3 with respect to all real subalgebras of $C \ell_{4}$. In turn, for the algebra $C \ell_{1,4}$ there exists a homomorphic mapping $\epsilon: C_{1,4} \rightarrow{ }^{\epsilon} C \ell_{4,0}$, where ${ }^{\epsilon} C_{4,0} \simeq C \ell_{4,0} /$ Ker $\epsilon$ is a quotient algebra, Ker $\epsilon$ is the kernel of the homomorphism $\epsilon$. It is shown that at the mapping $\epsilon$ the CPT group of the initial algebra is reduced to one from its subgroups. Discrete symmetries on the quotient representations of the algebra $C \ell_{1,4}$, associated with the space $\mathbb{R}^{1,4}$, are studied in the section 4 . The analogous situation takes place for the algebra $C_{3,2}$ associated with the anti-de Sitter space $\mathbb{R}^{3,2}$. In this case we have a decomposition $C \ell_{3,2} \simeq C \ell_{2,2} \oplus C \ell_{2,2}$, where $C \ell_{2,2} \simeq C \ell_{3,2}^{+}$is an even subalgebra of $C \ell_{3,2}$ (this decomposition is a particular case of the well-known isomorphism $C \ell_{p, q} \simeq$ $C \ell_{q, p-1} \oplus C \ell_{q, p-1}$, see, for example, [23]). Discrete symmetries of the quotient algebra ${ }^{\epsilon} \mathrm{Cl}_{2,2}$, obtained at the homomorphic mapping $\epsilon: C_{3,2} \rightarrow{ }^{\epsilon} C_{2,2}$, are considered in the section 5 .

\section{$2 \quad C P T$ group}

As is known, within the Clifford algebras there are infinitely many (continuous) automorphisms. Discrete symmetries $P$ and $T$ transform (reflect) space and time (two the most fundamental notions in physics), but in the Minkowski 4-dimensional spacetime continuum space and time are not separate and independent. For that reason a transformation of one (space or time) induces a transformation of another. Therefore, 
discrete symmetries should be expressed by such transformations of the continuum, which transform all its structure totally with a full preservation of discrete nature 1 . In 1949, Schouten [25] introduced such (discrete) automorphisms. In 1955, a first systematic description of these automorphisms was given by Rashevskii [26]. He showed that within the Clifford algebra $C_{p, q}$ over the real field $\mathbb{F}=\mathbb{R}$ there exist four fundamental automorphisms:

1) Identity: An automorphism $\mathcal{A} \rightarrow \mathcal{A}$ and $\mathbf{e}_{i} \rightarrow \mathbf{e}_{i}$.

This automorphism is an identical automorphism of the algebra $C_{p, q} . \mathcal{A}$ is an arbitrary element of $C \ell_{p, q}$.

2) Involution: An automorphism $\mathcal{A} \rightarrow \mathcal{A}^{\star}$ and $\mathbf{e}_{i} \rightarrow-\mathbf{e}_{i}$.

In more details, for an arbitrary element $\mathcal{A} \in C \ell_{p, q}$ there exists a decomposition $\mathcal{A}=\mathcal{A}^{\prime}+\mathcal{A}^{\prime \prime}$, where $\mathcal{A}^{\prime}$ is an element consisting of homogeneous odd elements, and $\mathcal{A}^{\prime \prime}$ is an element consisting of homogeneous even elements, respectively. Then the automorphism $\mathcal{A} \rightarrow \mathcal{A}^{\star}$ is such that the element $\mathcal{A}^{\prime \prime}$ is not changed, and the element $\mathcal{A}^{\prime}$ changes sign: $\mathcal{A}^{\star}=-\mathcal{A}^{\prime}+\mathcal{A}^{\prime \prime}$. If $\mathcal{A}$ is a homogeneous element, then

$$
\mathcal{A}^{\star}=(-1)^{k} \mathcal{A},
$$

where $k$ is a degree of the element. It is easy to see that the automorphism $\mathcal{A} \rightarrow \mathcal{A}^{\star}$ may be expressed via the volume element $\omega=\mathbf{e}_{12 \ldots p+q}$ :

$$
\mathcal{A}^{\star}=\omega \mathcal{A} \omega^{-1},
$$

where $\omega^{-1}=(-1)^{\frac{(p+q)(p+q-1)}{2}} \omega$. When $k$ is odd, the basis elements $\mathbf{e}_{i_{1} i_{2} \ldots i_{k}}$ the sign changes, and when $k$ is even, the sign is not changed.

3) Reversion: An antiautomorphism $\mathcal{A} \rightarrow \widetilde{\mathcal{A}}$ and $\mathbf{e}_{i} \rightarrow \mathbf{e}_{i}$.

The antiautomorphism $\mathcal{A} \rightarrow \widetilde{\mathcal{A}}$ is a reversion of the element $\mathcal{A}$, that is the substitution of each basis element $\mathbf{e}_{i_{1} i_{2} \ldots i_{k}} \in \mathcal{A}$ by the element $\mathbf{e}_{i_{k} i_{k-1} \ldots i_{1}}$ :

$$
\mathbf{e}_{i_{k} i_{k-1} \ldots i_{1}}=(-1)^{\frac{k(k-1)}{2}} \mathbf{e}_{i_{1} i_{2} \ldots i_{k}} .
$$

Therefore, for any $\mathcal{A} \in C_{p, q}$ we have

$$
\widetilde{\mathcal{A}}=(-1)^{\frac{k(k-1)}{2}} \mathcal{A}
$$

4) Conjugation: An antiautomorphism $\mathcal{A} \rightarrow \widetilde{\mathcal{A}^{\star}}$ and $\mathbf{e}_{i} \rightarrow-\mathbf{e}_{i}$.

This antiautomorphism is a composition of the antiautomorphism $\mathcal{A} \rightarrow \widetilde{\mathcal{A}}$ with the automorphism $\mathcal{A} \rightarrow \mathcal{A}^{\star}$. In the case of a homogeneous element from the formulae (1) and (3), it follows

$$
\widetilde{\mathcal{A}^{\star}}=(-1)^{\frac{k(k+1)}{2}} \mathcal{A} .
$$

\footnotetext{
${ }^{1}$ It is interesting to note that in the well-known Penrose twistor program 24 a spinor structure is understood as the underlying (more fundamental) structure with respect to Minkowski spacetime. In other words, spacetime continuum is not fundamental substance in the twistor approach, this is a fully derivative (in spirit of Leibnitz philosophy) entity generated by the underlying spinor structure. In this context spacetime discrete symmetries $P$ and $T$ should be considered as projections (shadows) of the fundamental automorphisms belonging to the background spinor structure.
} 
A finite group structure of the automorphism set $\{\mathrm{Id}, \star, \sim, \widetilde{\star}\}$ was studied in [21] with respect to discrete symmetries which compound PT group (so-called reflection group 2 .

Other important discrete symmetry is the charge conjugation $C$. In contrast with the transformations $P, T, P T$, the operation $C$ is not spacetime discrete symmetry. As is known, the Clifford algebra $C \ell_{n}$ over the complex field $\mathbb{F}=\mathbb{C}$ is associated with a complex vector space $\mathbb{C}^{n}$. Let $n=p+q$, then an extraction operation of the real subspace $\mathbb{R}^{p, q}$ in $\mathbb{C}^{n}$ forms the foundation of definition of the discrete transformation known in physics as a charge conjugation $C$. Indeed, let $\left\{\mathbf{e}_{1}, \ldots, \mathbf{e}_{n}\right\}$ be an orthobasis in the space $\mathbb{C}^{n}, \mathbf{e}_{i}^{2}=1$. Let us remain the first $p$ vectors of this basis unchanged, and other $q$ vectors multiply by the factor $i$. Then the basis

$$
\left\{\mathbf{e}_{1}, \ldots, \mathbf{e}_{p}, i \mathbf{e}_{p+1}, \ldots, i \mathbf{e}_{p+q}\right\}
$$

allows one to extract the subspace $\mathbb{R}^{p, q}$ in $\mathbb{C}^{n}$. Namely, for the vectors $\mathbb{R}^{p, q}$ we take the vectors of $\mathbb{C}^{n}$ which decompose on the basis (5) with real coefficients. In such a way we obtain a real vector space $\mathbb{R}^{p, q}$ endowed (in general case) with a non-degenerate quadratic form

$$
Q(x)=x_{1}^{2}+x_{2}^{2}+\ldots+x_{p}^{2}-x_{p+1}^{2}-x_{p+2}^{2}-\ldots-x_{p+q}^{2},
$$

where $x_{1}, \ldots, x_{p+q}$ are coordinates of the vector $\mathbf{x}$ in the basis (15). It is easy to see that the extraction of $\mathbb{R}^{p, q}$ in $\mathbb{C}^{n}$ induces an extraction of a real subalgebra $C \ell_{p, q}$ in $C \ell_{n}$. Therefore, any element $\mathcal{A} \in C_{n}$ can be unambiguously represented in the form

$$
\mathcal{A}=\mathcal{A}_{1}+i \mathcal{A}_{2}
$$

where $\mathcal{A}_{1}, \mathcal{A}_{2} \in \ell_{p, q}$. The one-to-one mapping

$$
\mathcal{A} \longrightarrow \overline{\mathcal{A}}=\mathcal{A}_{1}-i \mathcal{A}_{2}
$$

transforms the algebra $C \ell_{n}$ into itself with preservation of addition and multiplication operations for the elements $\mathcal{A}$; the operation of multiplication of the element $\mathcal{A}$ by the number transforms to an operation of multiplication by the complex conjugate number. Any mapping of $C_{n}$ satisfying these conditions is called a pseudoautomorphism. Thus, the extraction of the subspace $\mathbb{R}^{p, q}$ in the space $\mathbb{C}^{n}$ induces in the algebra $C \ell_{n}$ a pseudoautomorphism $\mathcal{A} \rightarrow \overline{\mathcal{A}}[26$, 27]. Compositions of $\mathcal{A} \rightarrow \overline{\mathcal{A}}$ with the fundamental automorphisms allow one to extend the set $\{\operatorname{Id}, \star, \sim \widetilde{\star}\}$ by the pseudoautomorphisms $\mathcal{A} \rightarrow \overline{\mathcal{A}}, \mathcal{A} \rightarrow \overline{\mathcal{A}^{\star}}, \mathcal{A} \rightarrow \overline{\widetilde{\mathcal{A}}}, \mathcal{A} \rightarrow \overline{\widetilde{\mathcal{A}^{\star}}}$ [22]. A finite group structure of an automorphism set $\{\mathrm{Id}, \star, \sim, \widetilde{\star},-\bar{\star}, \stackrel{\sim}{\sim}, \overline{\widetilde{\star}}\}$ was studied in [22] with respect to $C P T$ symmetries.

Further, in accordance with Wedderburn theorem any finite-dimensional associative simple algebra $\mathfrak{A}$ over the field $\mathbb{F}$ is isomorphic to a full matrix algebra $\operatorname{Mat}_{n}(\mathbb{K})$, where $n$ is natural number defined unambiguously, and $\mathbb{K}$ a division ring defined

\footnotetext{
${ }^{2}$ Some applications of the fundamental automorphisms to discrete symmetries of quantum field theory were considered by Rashevskii in [26] (see also his paper [27])
} 
with an accuracy of isomorphism. According to Wedderburn theorem the antiautomorphism $\mathcal{A} \rightarrow \widetilde{\mathcal{A}}$ corresponds to an antiautomorphism of the full matrix algebra $\operatorname{Mat}_{2^{m}}(\mathbb{K}): A \rightarrow A^{t}$, in virtue of the well-known relation $(A B)^{t}=B^{t} A^{t}$, where $t$ is a symbol of transposition, $n=2 m$. On the other hand, in the matrix representation of the elements $\mathcal{A} \in C \ell_{p, q}$, for the antiautomorphism $\mathcal{A} \rightarrow \widetilde{\mathcal{A}}$ we have $\mathrm{A} \rightarrow \widetilde{\mathrm{A}}$. $\mathrm{A}$ composition of the two antiautomorphisms, $\mathrm{A}^{t} \rightarrow \mathrm{A} \rightarrow \widetilde{\mathrm{A}}$, gives an automorphism $A^{t} \rightarrow \widetilde{A}$, which is an internal automorphism of the algebra $\operatorname{Mat}_{2^{m}}(\mathbb{K}):$

$$
\widetilde{\mathrm{A}}=\mathrm{EA}^{t} \mathrm{E}^{-1}
$$

where $\mathrm{E}$ is a matrix, by means of which the antiautomorphism $\mathcal{A} \rightarrow \widetilde{\mathcal{A}}$ is expressed in the matrix representation of the algebra $C \ell_{p, q}$. Under action of the antiautomorphism $\mathcal{A} \rightarrow \widetilde{\mathcal{A}}$ the units of $C \ell_{p, q}$ remain unaltered, $\mathbf{e}_{i} \rightarrow \mathbf{e}_{i}$; therefore in the matrix representation, we must demand $\mathcal{E}_{i} \rightarrow \mathcal{E}_{i}$, where $\mathcal{E}_{i}=\gamma\left(\mathbf{e}_{i}\right)$ also. Therefore, for the definition of the matrix $\mathrm{E}$ in accordance with (7) we have

$$
\mathcal{E}_{i} \longrightarrow \mathcal{E}_{i}=\mathrm{E}^{t} \mathrm{E}^{-1}
$$

A spinor representation of the automorphism $\mathcal{A} \rightarrow \mathcal{A}^{\star}$ follows directly from (2): $\mathrm{A}^{\star}=\mathrm{WAW}^{-1}$, where $\mathrm{W}=\mathcal{E}_{1} \mathcal{E}_{2} \cdots \mathcal{E}_{p+q}$. Further, a spinor representation of the pseudoautomorphism $\mathcal{A} \rightarrow \overline{\mathcal{A}}$ of the algebra $C \ell_{n}$ when $n \equiv 0(\bmod 2)$ is defined as follows. In the spinor representation the every element $\mathcal{A} \in C_{n}$ should be represented by some matrix $A$, and the pseudoautomorphism (6) takes a form of the pseudoautomorphism of the full matrix algebra $\mathrm{Mat}_{2^{n / 2}}$ :

$$
\mathrm{A} \longrightarrow \overline{\mathrm{A}} \text {. }
$$

On the other hand, a transformation replacing the matrix $A$ by the complex conjugate matrix, $\mathrm{A} \rightarrow \dot{\mathrm{A}}$, is also some pseudoautomorphism of the algebra $\mathrm{Mat}_{2^{n / 2}}$. The composition of the two pseudoautomorpisms $\dot{A} \rightarrow \mathrm{A}$ and $\mathrm{A} \rightarrow \overline{\mathrm{A}}, \dot{\mathrm{A}} \rightarrow \mathrm{A} \rightarrow \overline{\mathrm{A}}$, is an internal automorphism $\dot{A} \rightarrow \bar{A}$ of the full matrix algebra $\mathrm{Mat}_{2^{n / 2}}$ :

$$
\overline{\mathrm{A}}=\Pi \dot{\mathrm{A}} \Pi^{-1},
$$

where $\Pi$ is a matrix of the pseudoautomorphism $\mathcal{A} \rightarrow \overline{\mathcal{A}}$ in the spinor representation. The sufficient condition for definition of the pseudoautomorphism $\mathcal{A} \rightarrow \overline{\mathcal{A}}$ is a choice of the matrix $\Pi$ in such a way that the transformation $A \rightarrow \Pi \dot{A} \Pi^{-1}$ transfers into itself the matrices $\mathcal{E}_{1}, \ldots, \mathcal{E}_{p}, i \mathcal{E}_{p+1}, \ldots, i \mathcal{E}_{p+q}$ (the matrices of the spinbasis of $C \ell_{p, q}$ ), that is,

$$
\mathcal{E}_{i} \longrightarrow \mathcal{E}_{i}=\Pi \dot{\mathcal{E}}_{i} \Pi^{-1} \quad(i=1, \ldots, p+q) .
$$

The more detailed spinor representation of the pseudoautomorphism $\mathcal{A} \rightarrow \overline{\mathcal{A}}$ with respect to a division ring structure of the real subalgebras is given by the following theorem.

Theorem 1 ([22]). Let $C_{n}$ be a complex Clifford algebra for $n \equiv 0(\bmod 2)$ and let $C \ell_{p, q} \subset \mathbb{C}_{n}$ be its subalgebra with a real division ring $\mathbb{K} \simeq \mathbb{R}$ when $p-q \equiv 0,2(\bmod 8)$ 
and quaternionic division ring $\mathbb{K} \simeq \mathbb{H}$ when $p-q \equiv 4,6(\bmod 8), n=p+q$. Then in dependence on the division ring structure of the real subalgebra $C_{p, q}$ the matrix $\Pi$ of the pseudoautomorphism $\mathcal{A} \rightarrow \overline{\mathcal{A}}$ has the following form:

1) $\mathbb{K} \simeq \mathbb{R}, p-q \equiv 0,2(\bmod 8)$.

The matrix $\Pi$ for any spinor representation over the ring $\mathbb{K} \simeq \mathbb{R}$ is proportional to the unit matrix.

2) $\mathbb{K} \simeq \mathbb{H}, p-q \equiv 4,6(\bmod 8)$.

$\Pi=\mathcal{E}_{\alpha_{1} \alpha_{2} \cdots \alpha_{a}}$ when $a \equiv 0(\bmod 2)$ and $\Pi=\mathcal{E}_{\beta_{1} \beta_{2} \cdots \beta_{b}}$ when $b \equiv 1(\bmod 2)$, where $a$ complex matrices $\mathcal{E}_{\alpha_{t}}$ and b real matrices $\mathcal{E}_{\beta_{s}}$ form a basis of the spinor representation of the algebra $C_{p, q}$ over the ring $\mathbb{K} \simeq \mathbb{H}, a+b=p+q, 0<t \leq a, 0<s \leq b$. At this point,

$$
\begin{aligned}
& \Pi \dot{\Pi}=\mathrm{I} \quad \text { if } a, b \equiv 0,1 \quad(\bmod 4), \\
& \Pi \dot{\Pi}=-\mathrm{I} \quad \text { if } a, b \equiv 2,3 \quad(\bmod 4),
\end{aligned}
$$

where I is the unit matrix.

Spinor representations of the all other automorphisms from the $\operatorname{set}\{\operatorname{Id}, \star, \sim, \widetilde{\star},-\bar{\star}, \bar{\sim}, \overline{\widetilde{\star}}\}$ are defined in a similar manner. We list these transformations and their spinor representations:

$$
\begin{aligned}
\mathcal{A} \longrightarrow \mathcal{A}^{\star}, & \mathrm{A}^{\star}=\mathrm{WAW}^{-1}, \\
\mathcal{A} \longrightarrow \widetilde{\mathcal{A}}, & \widetilde{\mathcal{A}}=\mathrm{EA}^{t} \mathrm{E}^{-1}, \\
\mathcal{A} \longrightarrow \widetilde{\mathcal{A}^{\star}}, & \widetilde{\mathrm{A}^{\star}}=\mathrm{CA}^{t} \mathrm{C}^{-1}, \quad \mathrm{C}=\mathrm{EW}, \\
\mathcal{A} \longrightarrow \overline{\mathcal{A}}, & \overline{\mathrm{A}}=\Pi \mathrm{A}^{*} \Pi^{-1}, \\
\mathcal{A} \longrightarrow \overline{\mathcal{A}^{\star}}, & \overline{\mathrm{A}^{\star}}=\mathrm{KA}^{*} \mathrm{~K}^{-1}, \quad \mathrm{~K}=\Pi \mathrm{W}, \\
\mathcal{A} \longrightarrow \overline{\widetilde{\mathcal{A}}}, & \overline{\widetilde{\mathrm{A}}}=\mathrm{S}\left(\mathrm{A}^{t}\right)^{*} \mathrm{~S}^{-1}, \quad \mathrm{~S}=\Pi \mathrm{E}, \\
\mathcal{A} \longrightarrow \overline{\widetilde{\mathcal{A}^{\star}}}, & \overline{\widetilde{\mathrm{A}^{\star}}}=\mathrm{F}\left(\mathrm{A}^{*}\right)^{t} \mathrm{~F}^{-1}, \quad \mathrm{~F}=\Pi \mathrm{C},
\end{aligned}
$$

It is easy to verify that an automorphism set $\{\mathrm{Id}, \star, \sim, \widetilde{\star},-\bar{\mp}, \bar{\sim}, \overline{\widetilde{\star}}\}$ of $C \ell_{n}$ forms a finite group of order 8 .

Further, let $C \ell_{n}$ be a Clifford algebra over the field $\mathbb{F}=\mathbb{C}$ and let $\operatorname{CPT}\left(C \ell_{n}\right)=$ $\{\mathrm{Id}, \star, \sim, \widetilde{\star},-\bar{\star}, \bar{\sim}, \overline{\widetilde{\star}}\}$ be an automorphism group of the algebra $C \ell_{n}$. Then there is an isomorphism between $\mathrm{CPT}\left(C \ell_{n}\right)$ and a $C P T$ group of the discrete transformations, $\mathrm{CPT}\left(C \ell_{n}\right) \simeq\{1, P, T, P T, C, C P, C T, C P T\} \simeq \mathbb{Z}_{2} \times \mathbb{Z}_{2} \times \mathbb{Z}_{2}$. In this case, space inversion $P$, time reversal $T$, full reflection $P T$, charge conjugation $C$, transformations $C P, C T$ and the full $C P T$-transformation correspond to the automorphism $\mathcal{A} \rightarrow \mathcal{A}^{\star}$, antiautomorphisms $\mathcal{A} \rightarrow \widetilde{\mathcal{A}}, \mathcal{A} \rightarrow \widetilde{\mathcal{A}^{\star}}$, pseudoautomorphisms $\mathcal{A} \rightarrow \overline{\mathcal{A}}, \mathcal{A} \rightarrow \overline{\mathcal{A}^{\star}}$, pseudoantiautomorphisms $\mathcal{A} \rightarrow \overline{\widetilde{\mathcal{A}}}$ and $\mathcal{A} \rightarrow \overline{\widetilde{\mathcal{A}^{\star}}}$, respectively [22].

The group $\{1, P, T, P T, C, C P, C T, C P T\}$ at the conditions $P^{2}=T^{2}=$ $(P T)^{2}=C^{2}=(C P)^{2}=(C T)^{2}=(C P T)^{2}=1$ and commutativity of all the elements forms an Abelian group of order 8, which is isomorphic to a cyclic group $\mathbb{Z}_{2} \times \mathbb{Z}_{2} \times \mathbb{Z}_{2}$. In turn, the automorphism group $\{\operatorname{Id}, \star, \sim, \widetilde{\star},-\bar{\star}, \bar{\sim}, \overline{\widetilde{\star}}\}$ in virtue 


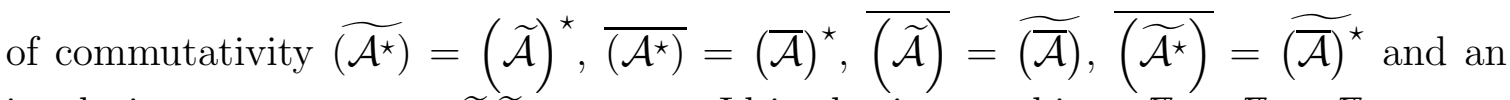
involution property $\star \star=\sim \sim \sim \sim-$ Id is also isomorphic to $\mathbb{Z}_{2} \times \mathbb{Z}_{2} \times \mathbb{Z}_{2}$ :

$$
\{1, P, T, P T, C, C P, C T, C P T\} \simeq\{\mathrm{Id}, \star, \sim, \widetilde{\star},-\bar{\star}, \bar{\sim}, \overline{\widetilde{\star}}\} \simeq \mathbb{Z}_{2} \times \mathbb{Z}_{2} \times \mathbb{Z}_{2}
$$

In 2003, the CPT group was introduced [22] in the context of an extension of automorphism groups of Clifford algebras. The relationship between $C P T$ groups and extraspecial groups and universal coverings of orthogonal groups was established in [22, 28]. In 2004, Socolovsky considered the CPT group of the spinor field with respect to phase factors [29]. CPT groups of spinor fields in the de Sitter spaces of different signatures were studied in the works [30, 31]. CPT groups for higher spin fields have been defined in [32] on the spinspaces associated with representations of the spinor group $\operatorname{Spin}_{+}(1,3)$ (a universal covering of the proper orthocronous Lorentz group).

\subsection{Salingaros groups}

As it has been shown previously, CPT groups have explicit realizations via the finite groups. As is known [33]-37], a structure of the Clifford algebras admits a very elegant description in terms of finite groups. In accordance with a multiplication rule

$$
\begin{gathered}
\mathbf{e}_{i}^{2}=\sigma(p-i) \mathbf{e}_{0}, \quad \mathbf{e}_{i} \mathbf{e}_{j}=-\mathbf{e}_{j} \mathbf{e}_{i}, \\
\sigma(n)= \begin{cases}-1 & \text { if } n \leq 0 \\
+1 & \text { if } n>0,\end{cases}
\end{gathered}
$$

basis elements of the Clifford algebra $C \ell_{p, q}$ (the algebra over the field of real numbers, $\mathbb{F}=\mathbb{R}$ ) form a finite group of order $2^{n+1}$,

$$
G(p, q)=\left\{ \pm 1, \pm \mathbf{e}_{i}, \pm \mathbf{e}_{i} \mathbf{e}_{j}, \pm \mathbf{e}_{i} \mathbf{e}_{j} \mathbf{e}_{k}, \ldots, \pm \mathbf{e}_{1} \mathbf{e}_{2} \cdots \mathbf{e}_{n}\right\} \quad(i<j<k<\ldots) .
$$

Salingaros showed [33, 34] that there exist five distinct types of finite groups (13) that arise from Clifford algebras. In [33, 34] they were called 'vee groups' and were labelled as

$$
N_{\text {odd }}, N_{\text {even }}, \Omega_{\text {odd }}, \Omega_{\text {even }}, S_{k}
$$

The odd $N$-groups correspond to real spinors, for example, $N_{1}$ is related to real 2-spinors, and $N_{3}$ is the group of the real Majorana matrices. The even $N$-groups define the quaternionic groups. The $S$-groups are the 'spinor groups' $\left(S_{k}=N_{2 k} \times \mathbb{C} \simeq\right.$ $\left.N_{2 k-1} \times \mathbb{C}\right): S_{1}$ is the group of the complex Pauli matrices, and $S_{2}$ is the group of the Dirac matrices. Furthermore, the $\Omega$-groups are double copies of the $N$-groups and can be written as a direct product of the $N$-groups with the group of two elements $\mathbb{Z}_{2}$ :

$$
\Omega_{k}=N_{k} \times \mathbb{Z}_{2}
$$


The five distinct types of Salingaros groups correspond to the five division rings of the real Clifford algebras [28]:

$$
\begin{aligned}
N_{\text {odd }} & \leftrightarrow \quad \ell_{p, q}, p-q \equiv 0,2 \quad(\bmod 8), \mathbb{K} \simeq \mathbb{R} ; \\
N_{\text {even }} & \leftrightarrow \quad \ell_{p, q}, p-q \equiv 4,6 \quad(\bmod 8), \mathbb{K} \simeq \mathbb{H} ; \\
\Omega_{\text {odd }} & \leftrightarrow \quad \ell_{p, q}, p-q \equiv 1 \quad(\bmod 8), \mathbb{K} \simeq \mathbb{R} \oplus \mathbb{R} ; \\
\Omega_{\text {even }} & \leftrightarrow \quad \ell_{p, q}, p-q \equiv 5 \quad(\bmod 8), \mathbb{K} \simeq \mathbb{H} \oplus \mathbb{H} ; \\
S_{k} & \leftrightarrow \quad \ell_{p, q}, p-q \equiv 3,7 \quad(\bmod 8), \mathbb{K} \simeq \mathbb{C} .
\end{aligned}
$$

Let $\mathbf{Z}(p, q) \subset C \ell_{p, q}$ be a center of the finite group (13). In such a way, we have three distinct realizations of the center $\mathbf{Z}(p, q)$ :

$$
\begin{aligned}
& \mathbf{Z}(p, q)=\{1,-1\} \simeq \mathbb{Z}_{2} \text { if } p-q \equiv 0,2,4,6 \quad(\bmod 8) \\
& \mathbf{Z}(p, q)=\{1,-1, \omega,-\omega\} \simeq \mathbb{Z}_{2} \times \mathbb{Z}_{2} \text { if } p-q \equiv 1,5 \quad(\bmod 8) \\
& \mathbf{Z}(p, q)=\{1,-1, \omega,-\omega\} \simeq \mathbb{Z}_{4} \text { if } p-q \equiv 3,7 \quad(\bmod 8)
\end{aligned}
$$

The Abelian groups $\mathbf{Z}(p, q)$ are the subgroups of the Salingaros groups (14). Namely, $N$-groups have the center $\mathbb{Z}_{2}, \Omega$-groups have the center $\mathbb{Z}_{2} \times \mathbb{Z}_{2}$, and $S$-group has the center $\mathbb{Z}_{4}$.

The following Theorem presents a key result in the group structure of $C \ell_{p, q}$.

Theorem 2 (Salingaros [33]). The factor group $G(p, q) / \mathbf{Z}(p, q)$ is the Abelian group $\left(\mathbb{Z}_{2}\right)^{\times 2 k}=\mathbb{Z}_{2} \times \mathbb{Z}_{2} \times \cdots \times \mathbb{Z}_{2}$ (2k times):

$$
\frac{G(p, q)}{\mathbf{Z}(p, q)}: \frac{N_{2 k-1}}{\mathbb{Z}_{2}} \simeq \frac{N_{2 k}}{\mathbb{Z}_{2}} \simeq \frac{\Omega_{2 k-1}}{\mathbb{Z}_{2} \times \mathbb{Z}_{2}} \simeq \frac{\Omega_{2 k}}{\mathbb{Z}_{2} \times \mathbb{Z}_{2}} \simeq \frac{S_{k}}{\mathbb{Z}_{4}} \simeq\left(\mathbb{Z}_{2}\right)^{\times 2 k} .
$$

This Theorem allows one to identify the Salingaros groups with extraspecial groups [33. 35, 36]. As known, a finite group $G$ is called an extraspecial 2-group if $G$ is of order $2^{n}$ and $G / \mathbf{Z}(G)$ is the Abelian group $\mathbb{Z}_{2} \times \cdots \times \mathbb{Z}_{2}(n-1$ times). Further, if $G$ is the extraspecial 2 -group of order $2^{2 k+1}$, then

$$
\begin{aligned}
& G \simeq D_{4} \circ \cdots \circ D_{4} \quad(k \text { times }), \text { or } \\
& G \simeq Q_{4} \circ D_{4} \circ \cdots \circ D_{4} \quad(k-1 \text { times }),
\end{aligned}
$$

where o means a central product of two groups: that is, the direct product with centers identified. For example, the direct product $Q_{4} \times D_{4}$ has the resulting group of order $8 \times 8=64$, and its center is the direct product of the two individual centers and is equal to $\mathbb{Z}_{2} \times \mathbb{Z}_{2}$. In contrast, the central product $Q_{4} \circ D_{4}$ amalgamates the $\mathbb{Z}_{2}$ center of $Q_{4}$ with the $\mathbb{Z}_{2}$ of $D_{4}$ to give the center of $Q_{4} \circ D_{4}$ as $\mathbb{Z}_{2}$. Therefore, the central product $Q_{4} \circ D_{4}$ is of order 32. In the case where one center is a subgroup of the other center, they both amalgamate into the larger center.

In terms of the extraspecial groups all the Salingaros groups take the form:

$$
\begin{aligned}
N_{2 k-1} & \simeq\left(N_{1}\right)^{\circ k} \\
N_{2 k} & \simeq N_{2} \circ\left(N_{1}\right)^{\circ(k-1)}, \\
\Omega_{2 k-1} & \simeq N_{2 k-1} \circ\left(\mathbb{Z}_{2} \times \mathbb{Z}_{2}\right), \\
\Omega_{2 k} & \simeq N_{2 k} \circ\left(\mathbb{Z}_{2} \times \mathbb{Z}_{2}\right), \\
S_{k} & \simeq N_{2 k-1} \circ \mathbb{Z}_{4} \simeq N_{2 k} \circ \mathbb{Z}_{4} .
\end{aligned}
$$


As is known, an important property of each finite group is its order structure. The order of a particular element $\alpha$ in the group is the smallest integer $p$ for which $\alpha^{p}=1$. For example, the Tab. 1 lists the number of distinct elements in each group which have order 2,4 , or 8 (the identity 1 is the only element of order 1 ).

\begin{tabular}{|c|c|c|c|c|}
\hline & \multirow[b]{2}{*}{ Type } & \multicolumn{3}{|c|}{ Order structure } \\
\hline & & 2 & 4 & 8 \\
\hline 1. $\mathbb{Z}_{2} \times \mathbb{Z}_{2} \times \mathbb{Z}_{2}$ & Abelian & 7 & & \\
\hline 2. $\mathbb{Z}_{4} \times \mathbb{Z}_{2}$ & & 3 & 4 & \\
\hline 3. $\mathbb{Z}_{8}$ & & 1 & 2 & 4 \\
\hline 4. $D_{4}$ & Non-Abelian & 5 & 2 & \\
\hline 5. $Q_{4}$ & & 1 & 6 & \\
\hline 6. $\stackrel{*}{\mathbb{Z}}_{4} \times \mathbb{Z}_{2}$ & & 3 & 4 & \\
\hline
\end{tabular}

Tab. 1: Finite groups of order 8 .

Following to Salingaros an order structure of the finite group is defined by the expression

$$
\left(o_{1}, o_{2}, o_{4}, o_{8}\right)
$$

where $o_{1}$ is the number of elements of order $1, o_{2}$ is the number of elements of order 2 and so on. Salingaros drops the first entry as it always 1 . Therefore, we will use below the order structure with three entries, $\left(o_{2}, o_{4}, o_{8}\right)$. Of course, $\mathbb{Z}_{8}$ does not occur as a $G(p, q)$ (Salingaros group), since every element of $G(p, q)$ has order 1,2 , or 4 . The groups $\mathbb{Z}_{4} \times \mathbb{Z}_{2}$ and $\stackrel{\mathbb{Z}}{4}_{4} \times \mathbb{Z}_{2}$ in Tab. 1 have the same order structure $(3,4,0)$, but the sequences of ' + ' and '-' are different in their signatures $(a, b, c, d, e, f, g)$. The group $\stackrel{\mathbb{Z}}{4}_{4} \times \mathbb{Z}_{2}$ is an non-Abelian analogue of $\mathbb{Z}_{4} \times \mathbb{Z}_{2}$.

\subsection{Dąbrowski groups}

In 1958, Shirokov pointed out [38, 39] that a universal covering of the inhomogeneous Lorentz group has eight inequivalent realizations. Later on, in the eighties this idea was applied to a general orthogonal group $O(p, q)$ by Dạbrowski [40]. As is known, the orthogonal group $O(p, q)$ of the real space $\mathbb{R}^{p, q}$ is represented by the semidirect product of a connected component $O_{0}(p, q)$ and a discrete subgroup $\{1, P, T, P T\}$. In general, there are eight double coverings of the orthogonal group $O(p, q)$ [40]:

$$
\rho^{a, b, c}: \operatorname{Pin}^{a, b, c}(p, q) \longrightarrow O(p, q),
$$

where $a, b, c \in\{+,-\}$. As is known, the group $O(p, q)$ consists of four connected components: identity connected component $O_{0}(p, q)$, and three components corresponding to space inversion $P$, time reversal $T$, and the combination of these two 
$P T$, that is, $O(p, q)=\left(O_{0}(p, q)\right) \cup P\left(Q_{0}(p, q)\right) \cup T\left(O_{0}(p, q)\right) \cup P T\left(O_{0}(p, q)\right)$. Further, since the four-element group (reflection group) $\{1, P, T, P T\}$ is isomorphic to the finite group $\mathbb{Z}_{2} \times \mathbb{Z}_{2}$, then $O(p, q)$ may be represented by a semidirect product $O(p, q) \simeq O_{0}(p, q) \odot\left(\mathbb{Z}_{2} \otimes \mathbb{Z}_{2}\right)$. The signs of $a, b, c$ correspond to the signs of the squares of the elements in $\operatorname{Pin}^{a, b, c}(p, q)$ which cover space inversion $P$, time reversal $T$ and a combination of these two $P T\left(a=-P^{2}, b=T^{2}, c=-(P T)^{2}\right.$ in Dạbrowski's notation [40] and $a=P^{2}, b=T^{2}, c=(P T)^{2}$ in Chamblin's notation [41] which we will use below). An explicit form of the group $\operatorname{Pin}^{a, b, c}(p, q)$ is given by the following semidirect product:

$$
\operatorname{Pin}^{a, b, c}(p, q) \simeq \frac{\left(\operatorname{Spin}_{+}(p, q) \odot C^{a, b, c}\right)}{\mathbb{Z}_{2}},
$$

where $C^{a, b, c}$ are the four double coverings of $\mathbb{Z}_{2} \times \mathbb{Z}_{2}$. All the eight universal coverings of the orthogonal group $O(p, q)$ are given in the Tab. 2. At this point, the group

\begin{tabular}{|c|l|l|}
\hline$a b c$ & $C^{a, b, c}$ & Remark \\
\hline+++ & $\mathbb{Z}_{2} \times \mathbb{Z}_{2} \times \mathbb{Z}_{2}$ & $P T=T P$ \\
+-- & $\mathbb{Z}_{2} \times \mathbb{Z}_{4}$ & $P T=T P$ \\
-+- & $\mathbb{Z}_{2} \times \mathbb{Z}_{4}$ & $P T=T P$ \\
--+ & $\mathbb{Z}_{2} \times \mathbb{Z}_{4}$ & $P T=T P$ \\
\hline--- & $Q_{4}$ & $P T=-T P$ \\
-++ & $D_{4}$ & $P T=-T P$ \\
+-+ & $D_{4}$ & $P T=-T P$ \\
++- & $D_{4}$ & $P T=-T P$ \\
\hline
\end{tabular}

Tab. 2: $P T$-structures.

$$
\operatorname{PT}\left(C \ell_{p, q}\right) \simeq \frac{C^{a, b, c}}{\mathbb{Z}_{2}}
$$

is the reflection group.

In turn, it has been shown 22] that there exist 64 universal coverings of the orthogonal group $O(p, q)$ :

$$
\rho^{a, b, c, d, e, f, g}: \operatorname{Pin}^{a, b, c, d, e, f, g} \longrightarrow O(p, q),
$$

where

$$
\operatorname{Pin}^{a, b, c, d, e, f, g}(p, q) \simeq \frac{\left(\operatorname{Spin}_{+}(p, q) \odot C^{a, b, c, d, e, f, g}\right)}{\mathbb{Z}_{2}}
$$

Here $C^{a, b, c, d, e, f, g}$ are five double coverings of the group $\mathbb{Z}_{2} \times \mathbb{Z}_{2} \times \mathbb{Z}_{2}$, and $a, b, c, d, e, f, g \in$ $\{+,-\}, a=P^{2}, b=T^{2}, c=(P T)^{2}, d=C^{2}, e=(C P)^{2}, f=(C T)^{2}, g=(C P T)^{2}$. All the possible double coverings $C^{a, b, c, d, e, f, g}$ are given in the Tab. 3. Thus, 


\begin{tabular}{|l|l|l|}
\hline a bc $d$ e fg & $C^{a, b, c, d, e, f, g}$ & Type \\
\hline+++++++ & $\mathbb{Z}_{2} \times \mathbb{Z}_{2} \times \mathbb{Z}_{2} \times \mathbb{Z}_{2}$ & Abelian \\
three '+' and four '-' & $\mathbb{Z}_{4} \times \mathbb{Z}_{2} \times \mathbb{Z}_{2}$ & \\
\hline one '+' and six '-' & $Q_{4} \times \mathbb{Z}_{2}$ & Non-Abelian \\
five '+' and two '-' & $D_{4} \times \mathbb{Z}_{2}$ & \\
three '+' and four '-', & $\mathbb{Z}_{4} \times \mathbb{Z}_{2} \times \mathbb{Z}_{2}$ & \\
\hline
\end{tabular}

Tab. 3: $C P T$-structures.

$$
C^{a, b, c, d, e, f, g}=\{ \pm 1, \pm P, \pm T, \pm P T, \pm C, \pm C P, \pm C T, \pm C P T\}
$$

is a full CPT group. $C^{a, b, c, d, e, f, g}$ is a finite group of order 16 (a complete classification of these groups is given in [22]). At this point, the group

$$
\operatorname{CPT}\left(C \ell_{p, q}\right)=\frac{C^{a, b, c, d, e, f, g}}{\mathbb{Z}_{2}}
$$

is called a generating group. It is easy to see that in case of the algebra $C_{p, q}$ (or subalgebra $\left.C \ell_{p, q} \subset C \ell_{n}\right)$ with the real division $\operatorname{ring} \mathbb{K} \simeq \mathbb{R}, p-q \equiv 0,2(\bmod 8), C P T$ structures, defined by the group (17), are reduced to the eight Shirokov-Dąbrowski $P T$-structures.

\section{$3 \quad C P T$ groups in the space $\mathbb{R}^{4,1}$}

In 1935, Dirac [42] introduced relativistic wave equations in a five-dimensional pseudoeuclidean space $\mathbb{R}^{4,1}$ (de Sitter space),

$$
\left(i \gamma_{0} \partial_{0}+i \gamma_{k} \partial_{k}-m\right) \psi=0
$$

or

$$
\left(i \gamma_{\mu} \partial_{\mu}+m\right) \psi=0
$$

where five $4 \times 4$ Dirac matrices $\gamma_{\mu}$ satisfy the relations

$$
\gamma_{\mu} \gamma_{\nu}+\gamma_{\nu} \gamma_{\mu}=2 g_{\mu \nu}, \quad \mu=0,1,2,3,4
$$

The algebra $C \ell_{4,1}$, associated with the space $\mathbb{R}^{4,1}$, has the type $p-q \equiv 3(\bmod 8)$. Hence it follows the isomorphism $C \ell_{4,1} \simeq C \ell_{4}$, where $C_{4}$ is a Clifford algebra over the complex field $\mathbb{F}=\mathbb{C}$ (so-called Dirac algebra).

A finite group $G(4,1)$ corresponding to $C \ell_{4,1} \simeq C \ell_{4}$ is a particular case of (13). So, in accordance with (13) the Dirac group $G(4,1)$ is defined by the following set:

$$
\begin{aligned}
G(4,1)=\left\{ \pm 1, \pm \mathbf{e}_{1}, \ldots, \pm \mathbf{e}_{5}, \pm \mathbf{e}_{1} \mathbf{e}_{2}, \ldots, \pm \mathbf{e}_{4} \mathbf{e}_{5}\right. \\
\left.\quad \pm \mathbf{e}_{1} \mathbf{e}_{2} \mathbf{e}_{3}, \ldots, \pm \mathbf{e}_{3} \mathbf{e}_{4} \mathbf{e}_{5}, \pm \mathbf{e}_{1} \mathbf{e}_{2} \mathbf{e}_{3} \mathbf{e}_{4}, \ldots, \pm \mathbf{e}_{2} \mathbf{e}_{3} \mathbf{e}_{4} \mathbf{e}_{5}, \pm \mathbf{e}_{1} \mathbf{e}_{2} \mathbf{e}_{3} \mathbf{e}_{4} \mathbf{e}_{5}\right\}
\end{aligned}
$$


It is a finite group of order 64 with the order structure $(31,32,0)$. The Dirac group is an extraspecial two-group. For this group the following isomorphism holds:

$$
G(4,1)=S_{2} \simeq N_{4} \circ \mathbb{Z}_{4} \simeq Q_{4} \circ D_{4} \circ \mathbb{Z}_{4}
$$

The center of $G(4,1)$ is isomorphic to the group $\mathbb{Z}_{4}$ (finite group of order 4 ). $G(4,1)$ is an non-Abelian group (as all Salingaros groups, except the first three groups $\mathbb{Z}_{2}$, $\Omega_{0}=\mathbb{Z}_{2} \times \mathbb{Z}_{2}$ and $\left.S_{0}=\mathbb{Z}_{4}\right)$.

In dependence on the division ring structure the algebra $C_{4}$ has five real subalgebras which correspond to real subspaces $\mathbb{R}^{p, q} \subset \mathbb{C}^{4}(p+q=4)$ with distinct signatures $(+,-,-,-),(-,+,+,+),(-,-,-,-),(+,+,+,+)$ and $(-,-,+,+)$. Three subalgebras with the quaternionic ring $\mathbb{K} \simeq \mathbb{H}$ : the spacetime algebra $C \ell_{1,3}, C \ell_{4,0}$ and $C \ell_{0,4}$. Two subalgebras with the real ring $\mathbb{K} \simeq \mathbb{R}$ : the Majorana $C \ell_{3,1}$ and $C \ell_{2,2}$ algebras. In accordance with (13) the each real subalgebra $C \ell_{p, q} \subset \mathbb{C}_{4}$ induces a finite group $G(p, q)$. Let us consider in detail the structure of these finite groups. Owing to (13), a spacetime group is defined by the followng set:

$$
\begin{aligned}
G(1,3)=\left\{ \pm 1, \pm \gamma_{0}, \pm \gamma_{1}, \pm \gamma_{2}, \pm \gamma_{3}, \pm \gamma_{0} \gamma_{1},\right. & \pm \gamma_{0} \gamma_{2} \\
\pm \gamma_{0} \gamma_{3}, \pm \gamma_{1} \gamma_{2}, \pm \gamma_{1} \gamma_{3}, \pm \gamma_{2} \gamma_{3}, & \pm \gamma_{0} \gamma_{1} \gamma_{2}, \pm \gamma_{0} \gamma_{1} \gamma_{3} \\
& \left. \pm \gamma_{0} \gamma_{2} \gamma_{3}, \pm \gamma_{1} \gamma_{2} \gamma_{3}, \pm \gamma_{0} \gamma_{1} \gamma_{2} \gamma_{3}\right\}
\end{aligned}
$$

It is a finite group of order 32 with the order structure $(11,20,0)$. Moreover, $G(1,3)$ is the extraspecial two-group. In Salingaros notation the following isomorphism holds:

$$
G(1,3)=N_{4} \simeq Q_{4} \circ D_{4}
$$

A center of the group $G(1,3)$ is isomorphic to a cyclic group $\mathbb{Z}_{2}$. $G(1,3)$ is the nonAbelian group which contains many subgroups both Abelian and non-Abelian. For example, the group of fundamental automorphisms of the algebra $C \ell_{1,3}$ is an Abelian subgroup of $G(1,3), \operatorname{PT}\left(C \ell_{1,3}\right)=\{\mathrm{Id}, \star, \sim, \widetilde{\star}\} \simeq\{1, P, T, P T\} \simeq \mathbb{Z}_{2} \times \mathbb{Z}_{2} \subset G(1,3)$. In turn, the automorphism group of the algebra $C \ell_{1,3}$ is a non-Abelian subgroup of $G(1,3), \operatorname{CPT}\left(C \ell_{1,3}\right) \simeq \subset G(1,3)$. Finite groups $G(4,0)$ and $G(0,4)$, corresponding to the subalgebras $C \ell_{4,0}$ and $C \ell_{0,4}$, are isomorphic to each other, since these groups possess the order structure $(11,20,0)$. This group isomorphism is a direct consequence of the algebra isomorphism $C \ell_{4,0} \simeq C \ell_{0,4}$. Therefore,

$$
G(4,0) \simeq G(0,4)=N_{4} \simeq Q_{4} \circ D_{4}
$$

The Majorana group $G(3,1)$ with the order structure $(19,12,0)$ is a central product of the two groups $D_{4}$ :

$$
G(3,1)=N_{3} \simeq N_{1} \circ N_{1} \simeq D_{4} \circ D_{4} .
$$

$G(3,1)$ is the non-Abelian group; a center of the group is isomorphic to $\mathbb{Z}_{2}$. The same isomorphism takes place for the group

$$
G(2,2)=N_{3} \simeq D_{4} \circ D_{4}
$$


Thus, the real subalgebras of the Dirac algebra $C \ell_{4}$ form five finite groups of order 32. Three subalgebras with the ring $\mathbb{K} \simeq \mathbb{H}$ form finite groups which isomorphic to the central product $Q_{4} \circ D_{4}$, and two subalgebras with the ring $\mathbb{K} \simeq \mathbb{R}$ form finite groups defined by the product $D_{4} \circ D_{4}$.

If we consider a spinor representation (a left regular representation in a spinspace $\mathbb{S})$, then the units $\mathbf{e}_{i}$ of the algebra $C \ell_{4}$ are replaced by $\gamma$-matrices via the rule $\gamma_{i}=$ $\gamma\left(\mathbf{e}_{i}\right)$, where $\gamma$ is a mapping of the form $C \ell_{p, q} \stackrel{\gamma}{\longrightarrow} \operatorname{End}_{\mathbb{K}}(\mathbb{S}), C \ell_{p, q} \subset C \ell_{4}$. In the papers [30, 31] matrices $\gamma_{i}=\gamma\left(\mathbf{e}_{i}\right)$ were defined via a Brauer-Weyl representation [43. Following to a more rigorous algebraic framework [44, we see that a definition procedure of the spinor representation over the ring $\mathbb{K}$ is hardly fixed and depends on the structure of primitive idempotents $f$ of the algebra $C \ell_{p, q}$. As is known, for the Clifford algebra $C \ell_{p, q}$ over the field $\mathbb{F}=\mathbb{R}$ there are isomorphisms $C \ell_{p, q} \simeq \operatorname{End}_{\mathbb{K}}\left(I_{p, q}\right) \simeq$ $\operatorname{Mat}_{2^{m}}(\mathbb{K})$, where $m=(p+q) / 2, I_{p, q}=C \ell_{p, q} f$ is a minimal left ideal of $C \ell_{p, q}$, and $\mathbb{K}=f C \ell_{p, q} f$ is a division ring of $C \ell_{p, q}$. A primitive idempotent of the algebra $C \ell_{p, q}$ has the form

$$
f=\frac{1}{2}\left(1 \pm \mathbf{e}_{\alpha_{1}}\right) \frac{1}{2}\left(1 \pm \mathbf{e}_{\alpha_{2}}\right) \cdots \frac{1}{2}\left(1 \pm \mathbf{e}_{\alpha_{k}}\right),
$$

where $\mathbf{e}_{\alpha_{1}}, \mathbf{e}_{\alpha_{2}}, \ldots, \mathbf{e}_{\alpha_{k}}$ are commuting elements with square 1 of the canonical basis of $C \ell_{p, q}$ generating a group of order $2^{k}$, that is, $\left(\mathbf{e}_{\alpha_{1}}, \mathbf{e}_{\alpha_{2}}, \ldots, \mathbf{e}_{\alpha_{k}}\right) \simeq\left(\mathbb{Z}_{2}\right)^{\times k}$, where $\left(\mathbb{Z}_{2}\right)^{\times k}=\mathbb{Z}_{2} \times \mathbb{Z}_{2} \times \cdots \times \mathbb{Z}_{2}$ ( $k$ times) is an Abelian group. The values of $k$ are defined by the formula $k=q-r_{q-p}$, where $r_{i}$ are the Radon-Hurwitz numbers [45, 46], values of which form a cycle of the period 8: $r_{i+8}=r_{i}+4$. The values of all $r_{i}$ are

\begin{tabular}{lllllllll}
$i$ & 0 & 1 & 2 & 3 & 4 & 5 & 6 & 7 \\
\hline$r_{i}$ & 0 & 1 & 2 & 2 & 3 & 3 & 3 & 3
\end{tabular}.

In terms of finite groups we have here an idempotent group $T_{p, q}(f) \simeq\left(\mathbb{Z}_{2}\right)^{\times(k+1)}$ of the order $2^{k+1}=2^{1+q-r_{q-p}}$. In turn, a quotient group $G(p, q) / T_{p, q}(f) \simeq G_{p, q}(f)$ is a finite group of order $2^{1+p+r_{q-p}}$ [47, 48, 49]. It is obvious that $G_{p, q}(f)$ is a normal subgroup of $G(p, q)$.

First of all, in accordance with Theorem 9 in [22] Clifford algebras over the field $\mathbb{F}=\mathbb{C}$ correspond to charged particles such as electron, proton and so on. CPT groups for the algebra $C \ell_{4,1} \simeq C \ell_{4}$ are considered in appendix.

In general case all the elements of $C^{a, b, c, d, e, f, g}\left(\operatorname{resp}\right.$. CPT $\left.\left(C \ell_{1,3}\right)\right)$ depend on the phase factors. Let us suppose

$$
P=\eta_{p} \mathrm{~W}, \quad T=\eta_{t} \mathrm{E}, \quad C=\eta_{c} \Pi,
$$

where $\eta_{p}, \eta_{t}, \eta_{c} \in \mathbb{C}^{*}=\mathbb{C}-\{0\}$. Taking into account (20), we obtain

$$
\begin{aligned}
& \mathrm{CPT}\left(C \ell_{1,3}\right) \simeq\{1, P, T, P T, C, C P, C T, C P T\} \\
& \simeq\left\{\mathbf{1}_{4}, \eta_{p} \mathrm{~W}, \eta_{t} \mathrm{E}, \eta_{p} \eta_{t} \mathrm{EW}, \eta_{c} \Pi, \eta_{c} \eta_{p} \Pi \mathrm{W}, \eta_{c} \eta_{t} \Pi \mathrm{E}, \eta_{c} \eta_{p} \eta_{t} \Pi \mathrm{EW}\right\} \simeq \\
& \quad \simeq\left\{\mathbf{1}_{4}, \eta_{p} \mathrm{~W}, \eta_{t} \mathrm{E}, \eta_{p} \eta_{t} \mathrm{C}, \eta_{c} \Pi, \eta_{c} \eta_{p} \mathrm{~K}, \eta_{c} \eta_{t} \mathrm{~S}, \eta_{c} \eta_{p} \eta_{t} \mathrm{~F}\right\}
\end{aligned}
$$

The multiplication table of this general group is given in Tab. 4. The Tab. 4 presents a general generating matrix for any possible CPT groups of the fields of any spin. 


\begin{tabular}{|c||c|c|c|c|c|c|c|c|}
\hline & $\mathbf{1}_{4}$ & $\eta_{p} \mathrm{~W}$ & $\eta_{t} \mathrm{E}$ & $\eta_{p t} \mathrm{C}$ & $\eta_{c} \Pi$ & $\eta_{c p} \mathrm{~K}$ & $\eta_{c t} \mathrm{~S}$ & $\eta_{c p t} \mathrm{~F}$ \\
\hline \hline $\mathbf{1}_{4}$ & $\mathbf{1}_{4}$ & $\eta_{p} \mathrm{~W}$ & $\eta_{t} \mathrm{E}$ & $\eta_{p t} \mathrm{C}$ & $\eta_{c} \Pi$ & $\eta_{c p} \mathrm{~K}$ & $\eta_{c t} \mathrm{~S}$ & $\eta_{c p t} \mathrm{~F}$ \\
\hline$\eta_{p} \mathrm{~W}$ & $\eta_{p} \mathrm{~W}$ & $\eta_{p}^{2} \mathrm{~W}^{2}$ & $\eta_{p t} \mathrm{WE}$ & $\eta_{p}^{2} \eta_{t} \mathrm{WC}$ & $\eta_{p c} \mathrm{~W} \Pi$ & $\eta_{c} \eta_{p}^{2} \mathrm{WK}$ & $\eta_{c p t} \mathrm{WS}$ & $\eta_{c t} \eta_{p}^{2} \mathrm{WF}$ \\
\hline$\eta_{t} \mathrm{E}$ & $\eta_{t} \mathrm{E}$ & $\eta_{p t} \mathrm{EW}$ & $\eta_{t}^{2} \mathrm{E}^{2}$ & $\eta_{p} \eta_{t}^{2} \mathrm{EC}$ & $\eta_{c t} \mathrm{E} \Pi$ & $\eta_{c p t} \mathrm{EK}$ & $\eta_{c} \eta_{t}^{2} \mathrm{ES}$ & $\eta_{c p} \eta_{t}^{2} \mathrm{EF}$ \\
\hline$\eta_{p t} \mathrm{C}$ & $\eta_{p t} \mathrm{C}$ & $\eta_{p}^{2} \eta_{t} \mathrm{CW}$ & $\eta_{p} \eta_{t}^{2} \mathrm{CE}$ & $\eta_{p t}^{2} \mathrm{C}^{2}$ & $\eta_{c p t} \mathrm{C} \Pi$ & $\eta_{c t} \eta_{p}^{2} \mathrm{CK}$ & $\eta_{c p} \eta_{t}^{2} \mathrm{CS}$ & $\eta_{c} \eta_{p t}^{2} \mathrm{CF}$ \\
\hline$\eta_{c} \Pi$ & $\eta_{c} \Pi$ & $\eta_{c p} \Pi \mathrm{W}$ & $\eta_{c t} \Pi \mathrm{E}$ & $\eta_{c p t} \Pi \mathrm{C}$ & $\eta_{c}^{2} \Pi^{2}$ & $\eta_{c}^{2} \eta_{p} \Pi \mathrm{K}$ & $\eta_{c}^{2} \eta_{t} \Pi \mathrm{S}$ & $\eta_{c}^{2} \eta_{p t} \Pi \mathrm{FF}$ \\
\hline$\eta_{c p} \mathrm{~K}$ & $\eta_{c p} \mathrm{~K}$ & $\eta_{c} \eta_{p}^{2} \mathrm{KW}$ & $\eta_{c p t} \mathrm{KE}$ & $\eta_{c t} \eta_{p}^{2} \mathrm{KC}$ & $\eta_{c}^{2} \eta_{p} \mathrm{~K} \Pi$ & $\eta_{c p}^{2} \mathrm{~K}^{2}$ & $\eta_{c}^{2} \eta_{p t} \mathrm{KS}$ & $\eta_{c p}^{2} \eta_{t} \mathrm{KF}$ \\
\hline$\eta_{c t} \mathrm{~S}$ & $\eta_{c t} \mathrm{~S}$ & $\eta_{c p t} \mathrm{SW}$ & $\eta_{c} \eta_{t}^{2} \mathrm{SE}$ & $\eta_{c p} \eta_{t}^{2} \mathrm{SC}$ & $\eta_{c}^{2} \eta_{t} \mathrm{~S} \Pi$ & $\eta_{c}^{2} \eta_{p t} \mathrm{SK}$ & $\eta_{c t}^{2} \mathrm{~S}^{2}$ & $\eta_{c t}^{2} \eta_{p} \mathrm{SF}$ \\
\hline$\eta_{c p t} \mathrm{~F}$ & $\eta_{c p t} \mathrm{~F}$ & $\eta_{c t} \eta_{p}^{2} \mathrm{FW}$ & $\eta_{c p} \eta_{t}^{2} \mathrm{FE}$ & $\eta_{c} \eta_{p t}^{2} \mathrm{FC}$ & $\eta_{c}^{2} \eta_{p t} \mathrm{~F} \Pi$ & $\eta_{c p}^{2} \eta_{t} \mathrm{FK}$ & $\eta_{c t}^{2} \eta_{p} \mathrm{FS}$ & $\eta_{c p t}^{2} \mathrm{~F}^{2}$ \\
\hline
\end{tabular}

Tab. 4: The multiplication table of general generating group $\mathrm{CPT}\left(C_{1,3}\right)$.

However, for the spinor field $\boldsymbol{\xi}^{\alpha}$ (spin-1/2 field) there are some restrictions. As is known, many textbooks on quantum field theory (see, for example, [51, 52]) state that a fermion and its associated antifermion have opposite relative intrinsic parity, that is, for the field of type $(1 / 2,0) \oplus(0,1 / 2)$ charge conjugation $C$ and space inversion $P$ anticommute, $C P=-P C,\{C, P\}=0$. At first glance, for higher spin fields, defined via tensor products $\boldsymbol{\xi}^{\alpha_{1} \alpha_{2} \ldots \alpha_{k}}=\sum \boldsymbol{\xi}^{\alpha_{1}} \otimes \boldsymbol{\xi}^{\alpha_{2}} \otimes \cdots \otimes \boldsymbol{\xi}^{\alpha_{k}}$, these restrictions should be changed. Hence it follows that bosonic and fermionic CPT groups should be different. Indeed, a boson and its associated antiboson carry same relative intrinsic parity, that is, for the field of type $(1,0) \oplus(0,1)$ the operations $C$ and $P$ commute, $C P=P C$, $[C, P]=0$, see [13]. However, Wigner [53] showed that there are theories where a fermion and its associated antifermion have the same relative intrinsic parity, $[C, P]=$ 0 ; and that a boson and its associated antiboson carry opposite relative intrinsic parity, $\{C, P\}=0$. Later on such theories were called as Bargmann-Wightman-Wigner-type quantum field theories [54, 55], see also [56, 57, 58] and references therein. At present day this ambiguous situation is known as ' $[C, P]_{ \pm}=0$ dilemma' [57].

In context of the present theory of discrete symmetries ' $[C, P]_{ \pm}=0$ dilemma' has a simple algebraic solution. It is obvious that a choice $[C, P]_{ \pm}=0$ depends on the spinor representations of $P=\eta_{p} \mathrm{~W}$ and $C=\eta_{c} \Pi$. In general, the matrix $\Pi$ has two different forms: $\Pi=\gamma_{\alpha_{1}} \gamma_{\alpha_{2}} \cdots \gamma_{\alpha_{a}}$ when $a \equiv 0(\bmod 2)$ and $\Pi=\gamma_{\beta_{1}} \gamma_{\beta_{2}} \cdots \gamma_{\beta_{b}}$ when $b \equiv 1(\bmod 2)$, where the complex matrices $\gamma_{\alpha_{t}}$ and the real matrices $\gamma_{\beta_{s}}$ form a basis of the spinor representation of the algebra $C \ell_{p, q}$ over the ring $\mathbb{K} \simeq \mathbb{H}, a+b=p+q$, $0<t \leq a, 0<s \leq b\left[22\right.$. It is easy to verify that $\Pi=\gamma_{\alpha_{1}} \gamma_{\alpha_{2}} \cdots \gamma_{\alpha_{a}}$ always commutes with $\mathbf{W}$, therefore, we have $[C, P]=0$ in this case. In turn, $\Pi=\gamma_{\beta_{1}} \gamma_{\beta_{2}} \cdots \gamma_{\beta_{b}}$ always anticommutes with $\mathrm{W}$, therefore, $\{C, P\}=0$. Among the groups CPT $\left(C_{1,3}\right)$, considered in this subsection, we see that $\mathrm{CPT}_{1}^{+}\left(C_{1,3}\right)$ with $\Pi=\gamma_{23}, \mathrm{CPT}_{2}^{+}\left(C \ell_{1,3}\right)$ and $\mathrm{CPT}_{3}^{+}\left(C \ell_{1,3}\right)$ with $\Pi=\gamma_{34}$ and $\mathrm{CPT}_{4}^{+}\left(C \ell_{1,3}\right)$ with $\Pi=\gamma_{24}$ lead to $[C, P]=0$. In contrast, $\mathrm{CPT}_{5}^{+}\left(C \ell_{1,3}\right)$ with $\Pi=\gamma_{1}$ leads to $\{C, P\}=0$. 


\section{$4 C P T$ groups in the space $\mathbb{R}^{1,4}$}

In the work [59] discrete symmetries for the spinor field in the de Sitter space with the signature $(+,-,-,-,-)$, that is, in the space $\mathbb{R}^{1,4}$, have been derived via the analysis of a de Sitter-Dirac wave equation. Discrete symmetries in the de Sitter space with the signature $(+,+,+,+,-)$, that is, in the space $\mathbb{R}^{4,1}$, have been considered in the previous section within an algebraic framework based on the automorphism set of Clifford algebras. In this section we study group structure of discrete transformations in the space $\mathbb{R}^{1,4}$.

In this context the de Sitter spacetime is understood as a hyperboloid embedded in the space $\mathbb{R}^{1,4}$ :

$$
X_{H}=\left\{x \in \mathbb{R}^{1,4}: x^{2}=\eta_{\alpha \beta} x^{\alpha} x^{\beta}=-H^{-2}\right\}, \quad \alpha, \beta=0,1,2,3,4,
$$

where $\eta_{\alpha \beta}=\operatorname{diag}(1,-1,-1,-1,-1)$.

The spinor wave equation in the Sitter spacetime (21) has been derived in [7, 59]. This equation has the form

$$
(-i \not x \gamma \cdot \bar{\partial}+2 i+\nu) \psi(x)=0
$$

where $\not x=\eta^{\alpha \beta} \gamma_{\alpha} x_{\beta}$ and $\bar{\partial}_{\alpha}=\partial_{\alpha}+H^{2} x_{\alpha} x \cdot \partial$. In this case $4 \times 4$ matrices $\gamma_{\alpha}$ are spinor representations of $C \ell_{1,4}$ and satisfy the relations

$$
\gamma_{\alpha} \gamma_{\beta}+\gamma_{\beta} \gamma_{\alpha}=2 \eta_{\alpha \beta} \mathbf{1}_{4}
$$

The finite group $G(1,4)$, associated with the algebra $C_{1,4}$, is a particular case of (13). It is a finite group of order 64 with the order structure $(23,40,0)$. In Salingaros notation we have the following isomorphism:

$$
G(1,4)=\Omega_{4} \simeq N_{4} \circ\left(\mathbb{Z}_{2} \times \mathbb{Z}_{2}\right) \simeq Q_{4} \circ D_{4} \circ\left(\mathbb{Z}_{2} \times \mathbb{Z}_{2}\right)
$$

Let us define $C P T$ group for the spinor field in the space $\mathbb{R}^{1,4}$. First of all, the transformation $C$ (the pseudoautomorphism $\mathcal{A} \rightarrow \overline{\mathcal{A}}$ ) for the algebras $C_{p, q}$ over the field $\mathbb{F}=\mathbb{R}$ and the ring $\mathbb{K} \simeq \mathbb{H}$ (the types $p-q \equiv 4,6(\bmod 8))$ corresponds to particle-antiparticle interchange $C^{\prime}$ (see [22, 28]). As is known, neutral particles are described within real representations of the Lorentz group. There are two classes of neutral particles: 1) particles which have antiparticles such as neutrons, neutrinos and so on; 2) particles which coincide with their antiparticles (for example, photons). The first class is described by the the algebras $C \ell_{p, q}$ over the field $\mathbb{F}=\mathbb{R}$ with the rings $\mathbb{K} \simeq \mathbb{H}$ and $\mathbb{K} \simeq \mathbb{H} \oplus \mathbb{H}($ the types $p-q \equiv 4,6(\bmod 8)$ and $p-q \equiv 5(\bmod 8))$, and the second class (truly neutral particles) is described by the algebras $C \ell_{p, q}$ over the field $\mathbb{F}=\mathbb{R}$ with the rings $\mathbb{K} \simeq \mathbb{R}$ and $\mathbb{K} \simeq \mathbb{R} \oplus \mathbb{R}$ (the types $p-q \equiv 0,2$ $(\bmod 8)$ and $p-q \equiv 1(\bmod 8))($ for more details see $[22,28,60])$.

Since $C \ell_{1,4}$ is a semisimple algebra over the field $\mathbb{F}=\mathbb{R}$, then for $C \ell_{1,4}$ we have the following decomposition: $C \ell_{1,4} \simeq C \ell_{1,4}^{+} \oplus C \ell_{1,4}^{+}$, where $C \ell_{1,4}^{+}$is an even subalgebra of 
$C \ell_{1,4}$. Moreover, there is an isomorphism $C \ell_{1,4}^{+} \simeq C \ell_{4,0}$, therefore, $C \ell_{1,4} \simeq C \ell_{4,0} \oplus C \ell_{4,0}$. This decomposition can be represented by the following scheme:

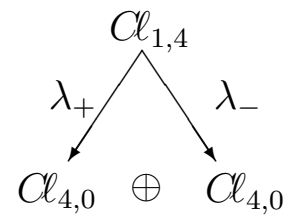

Here central idempotents

$$
\lambda_{+}=\frac{1+\mathbf{e}_{1} \mathbf{e}_{2} \mathbf{e}_{3} \mathbf{e}_{4} \mathbf{e}_{5}}{2}, \quad \lambda_{-}=\frac{1-\mathbf{e}_{1} \mathbf{e}_{2} \mathbf{e}_{3} \mathbf{e}_{4} \mathbf{e}_{5}}{2}
$$

satisfy the relations $\left(\lambda_{+}\right)^{2}=\lambda_{+},\left(\lambda_{-}\right)^{2}=\lambda_{-}, \lambda_{+} \lambda_{-}=0$. In accordance with 61] the idempotents $\lambda_{+}$and $\lambda_{-}$can be identified with helicity projection operators which distinguish left and right handed spinors.

The decomposition $C \ell_{1,4} \simeq C \ell_{4,0} \oplus C \ell_{4,0}$ induces a left-regular spinor representation $C \ell_{1,4} \stackrel{\gamma}{\longrightarrow} \operatorname{End}_{\mathbb{H} \oplus \hat{\mathbb{H}}}\left(\mathbb{S}_{2} \oplus \hat{\mathbb{S}}_{2}\right)$, where $\mathbb{S}_{2}(\mathbb{H}) \simeq I_{4,0}=C \ell_{4,0} f$ is a minimal left ideal of the subalgebra $C \ell_{4,0}, f$ is a primitive idempotent of $C \ell_{4,0}$. This spinor representation is realized within the matrix algebra ${ }^{2} \operatorname{Mat}_{2}(\mathbb{H})=\operatorname{Mat}_{2}(\mathbb{H}) \oplus \operatorname{Mat}_{2}(\mathbb{H})$.

However, there is a homomorphic mapping

$$
\epsilon: C \ell_{1,4} \longrightarrow{ }^{\epsilon} C_{4,0},
$$

where

$$
{ }^{\epsilon} \mathrm{Cl}_{4,0} \simeq \mathrm{Cl}_{4,0} / \mathrm{Ker} \epsilon
$$

is a quotient algebra, $\operatorname{Ker} \epsilon=\left\{\mathcal{A}^{1}-\omega \mathcal{A}^{1}\right\}$ is a kernel of the homomorphism $\epsilon$ (a bilateral ideal of the algebra $C \ell_{1,4}$, see [28]), $\mathcal{A}^{1} \in C_{4,0}, \omega=\mathbf{e}_{12345}$ is a volume element of $C \ell_{1,4}$. Under action of the homomorphism $\epsilon$ we cannot to transfer all the automorphisms of the initial algebra $C \ell_{1,4}$ onto the quotient algebra ${ }^{\epsilon} C_{4,0}$. So, in accordance with Theorem 14 in [28] at the mapping $\epsilon: C \ell_{1,4} \rightarrow{ }^{\epsilon} C \ell_{4,0}$ we can transfer onto ${ }^{\epsilon} C \ell_{4,0}$ only the antiautomorphism $\mathcal{A} \rightarrow \widetilde{\mathcal{A}}$, pseudoautomorphism $\mathcal{A} \rightarrow \overline{\mathcal{A}}$ (particle-antiparticle interchange $C^{\prime}$ ) and pseudoantiautomorphism $\mathcal{A} \rightarrow \overline{\widetilde{\mathcal{A}}}$. All other automorphisms of $C \ell_{1,4}$ do not transferred from $C \ell_{1,4}$ to ${ }^{\epsilon} C \ell_{4,0}$. Therefore, we have $\mathrm{PT}\left({ }^{\epsilon} C \ell_{4,0}\right)=\left\{1, T, C^{\prime}, C^{\prime} T\right\} \sim\{\mathrm{I}, \mathrm{E}, \Pi, \mathrm{S}\}$. It is easy to see that $\left\{1, T, C^{\prime}, C^{\prime} T\right\} \sim$ $\{\mathrm{I}, \mathrm{E}, \Pi, \mathrm{S}\}$ is the subgroup of the group $\mathrm{CPT}\left(C_{4,0}\right)$ considered in the subsection 3.2. For the idempotents $f_{1}^{ \pm}, f_{2}^{ \pm}, f_{3}^{ \pm}, f_{4}^{ \pm} \in C_{4,0}$, which generate the group (A.3), we obtain

$$
\mathrm{PT}\left({ }^{\epsilon} C_{4,0}\right) \simeq\left\{1, T, C^{\prime}, C^{\prime} T\right\} \simeq\left\{\mathbf{1}_{4}, \gamma_{34}, \eta_{c} \gamma_{34}, \eta_{c} \mathbf{1}_{4}\right\}
$$

The multiplication table of this group is shown in the Tab. 5. In both cases $\eta_{c}^{2}=1$ and $\eta_{c}^{2}=-1$ we have here an Abelian group PT $\left({ }^{\epsilon} C \ell_{4,0}\right) \simeq \mathbb{Z}_{4}$ and, therefore, $C^{\prime} P T$ groups (more precisely, $C^{\prime} T$ groups) are isomorphic to $C^{-,-,+} \simeq C^{-,+,-} \simeq \mathbb{Z}_{4} \times \mathbb{Z}_{2}$.

Further, for the idempotent $f_{5}^{+}=\frac{1}{2}\left(1+\mathbf{e}_{1234}\right) \in C_{4,0}$, which generates the group (A.5), we have

$$
\mathrm{PT}_{5}^{+}\left({ }^{\epsilon} C \ell_{4,0}\right) \simeq\left\{1, T, C^{\prime}, C^{\prime} T\right\} \simeq\left\{\mathbf{1}_{4}, \gamma_{1}, \eta_{c} \gamma_{1}, \eta_{c} \mathbf{1}_{4}\right\}
$$




\begin{tabular}{|c||c|c|c|c|}
\hline & 1 & $T$ & $C^{\prime}$ & $C^{\prime} T$ \\
\hline \hline 1 & 1 & $T$ & $C^{\prime}$ & $C^{\prime} T$ \\
\hline$T$ & $T$ & -1 & $-C^{\prime} T$ & $C^{\prime}$ \\
\hline$C^{\prime}$ & $C^{\prime}$ & $-C^{\prime} T$ & $-\left(C^{\prime}\right)^{2}$ & $\left(C^{\prime}\right)^{2} T$ \\
\hline$C^{\prime} T$ & $C^{\prime} T$ & $C^{\prime}$ & $\left(C^{\prime}\right)^{2} T$ & $\left(C^{\prime} T\right)^{2}$ \\
\hline
\end{tabular}

\begin{tabular}{|c||c|c|c|c|}
\hline & $\mathbf{1}_{4}$ & $\gamma_{34}$ & $\eta_{c} \gamma_{34}$ & $\eta_{c} \mathbf{1}_{4}$ \\
\hline \hline $\mathbf{1}_{4}$ & $\mathbf{1}_{4}$ & $\gamma_{34}$ & $\eta_{c} \gamma_{34}$ & $\eta_{c} \mathbf{1}_{4}$ \\
\hline$\gamma_{34}$ & $\gamma_{34}$ & $-\mathbf{1}_{4}$ & $-\eta_{c} \mathbf{1}_{4}$ & $\eta_{c} \gamma_{34}$ \\
\hline$\eta_{c} \gamma_{34}$ & $\eta_{c} \gamma_{34}$ & $-\eta_{c} \mathbf{1}_{4}$ & $-\eta_{c}^{2} \mathbf{1}_{4}$ & $\eta_{c}^{2} \gamma_{34}$ \\
\hline$\eta_{c} \mathbf{1}_{4}$ & $\eta_{c} \mathbf{1}_{4}$ & $\eta_{c} \gamma_{34}$ & $\eta_{c}^{2} \gamma_{34}$ & $\eta_{c}^{2} \mathbf{1}_{4}$ \\
\hline
\end{tabular}

Tab. 5: The multiplication table of $\mathrm{PT}\left({ }^{\epsilon} C_{4,0}\right) \simeq$ $\mathbb{Z}_{4}$ in dependence of the sign of $\eta_{c}^{2}$. $\mathbb{Z}_{4}$ with the signature $(-,-,+)$ at $\eta_{c}^{2}=1$ and $\mathbb{Z}_{4}$ with $(-,+,-)$ at $\eta_{c}^{2}=-1$.

\begin{tabular}{|c||c|c|c|c|}
\hline & 1 & $T$ & $C^{\prime}$ & $C^{\prime} T$ \\
\hline \hline 1 & 1 & $T$ & $C^{\prime}$ & $C^{\prime} T$ \\
\hline$T$ & $T$ & 1 & $C^{\prime} T$ & $C^{\prime}$ \\
\hline$C^{\prime}$ & $C^{\prime}$ & $C^{\prime} T$ & $\left(C^{\prime}\right)^{2}$ & $\left(C^{\prime}\right)^{2} T$ \\
\hline$C^{\prime} T$ & $C^{\prime} T$ & $C^{\prime}$ & $\left(C^{\prime}\right)^{2} T$ & $\left(C^{\prime} T\right)^{2}$ \\
\hline
\end{tabular}

\begin{tabular}{|c||c|c|c|c|}
\hline & $\mathbf{1}_{4}$ & $\gamma_{1}$ & $\eta_{c} \gamma_{1}$ & $\eta_{c} \mathbf{1}_{4}$ \\
\hline \hline $\mathbf{1}_{4}$ & $\mathbf{1}_{4}$ & $\gamma_{1}$ & $\eta_{c} \gamma_{1}$ & $\eta_{c} \mathbf{1}_{4}$ \\
\hline$\gamma_{1}$ & $\gamma_{1}$ & $\mathbf{1}_{4}$ & $\eta_{c} \mathbf{1}_{4}$ & $\eta_{c} \gamma_{1}$ \\
\hline$\eta_{c} \gamma_{1}$ & $\eta_{c} \gamma_{1}$ & $\eta_{c} \mathbf{1}_{4}$ & $\eta_{c}^{2} \mathbf{1}_{4}$ & $\eta_{c}^{2} \gamma_{1}$ \\
\hline$\eta_{c} \mathbf{1}_{4}$ & $\eta_{c} \mathbf{1}_{4}$ & $\eta_{c} \gamma_{1}$ & $\eta_{c}^{2} \gamma_{1}$ & $\eta_{c}^{2} \mathbf{1}_{4}$ \\
\hline
\end{tabular}

Tab. 6: The multiplication table of $\mathrm{PT}_{5}^{+}\left({ }^{\epsilon} C_{4,0}\right)$ in dependence on the sign of $\eta_{c}^{2}$. $\mathrm{PT}_{5}^{+}\left({ }^{\epsilon} C \ell_{4,0}\right) \simeq$ $\mathbb{Z}_{2} \times \mathbb{Z}_{2}$ with the signature $(+,+,+)$ at $\eta_{c}^{2}=1$ and $\mathrm{PT}_{5}^{+}\left({ }^{\epsilon} C_{4,0}\right) \simeq \mathbb{Z}_{4}$ with $(+,-,-)$ at $\eta_{c}^{2}=-1$.

The multiplication table of this group is given in Tab. 6 . In this case when $\eta_{c}^{2}=1$ we have the group $\mathrm{PT}_{5}^{+}\left({ }^{\epsilon} C \ell_{4,0}\right) \simeq \mathbb{Z}_{2} \times \mathbb{Z}_{2}$ and the group $\mathrm{PT}_{5}^{+}\left({ }^{\epsilon} C \ell_{4,0}\right) \simeq \mathbb{Z}_{4}$ when $\eta_{c}^{2}=-1$. In the first case we come to $C^{+,+,+} \simeq \mathbb{Z}_{2} \times \mathbb{Z}_{2} \times \mathbb{Z}_{2}$ and to $C^{+,-,-} \simeq \mathbb{Z}_{4} \times \mathbb{Z}_{2}$ in the second case.

\section{$5 \quad C P T$ groups in anti-de Sitter space $\mathbb{R}^{3,2}$}

As it has been shown in the section 4 , a five-dimensional pseudoeuclidean space $\mathbb{R}^{1,4}$ (so called de Sitter space) is associated with the algebra $C \ell_{1,4}$. In turn, the anti-de Sitter space $\mathbb{R}^{3,2}$, associated with the algebra $C l_{3,2}$, leads to the following extraspecial group of order 64 :

$$
G(3,2)=\Omega_{3} \simeq N_{3} \circ D_{2} \simeq D_{4} \circ D_{4} \circ D_{2}
$$

Let us study discrete symmetries arising from the algebra $C l_{3,2}$ associated with the space $\mathbb{R}^{3,2}$. First of all, $C_{3,2}$ is a semisimple algebra over the field $\mathbb{F}=\mathbb{R}$ with a double division $\operatorname{ring} \mathbb{K} \simeq \mathbb{R} \oplus \mathbb{R}$, the type $p-q \equiv 1(\bmod 8)$. In accordance with Theorem 9 in [22] for the algebras $C \ell_{p, q}$ over the real field $\mathbb{F}=\mathbb{R}$ with real division rings $\mathbb{K} \simeq \mathbb{R}$ 
and $\mathbb{K} \simeq \mathbb{R} \oplus \mathbb{R}$ (types $p-q \equiv 0,1,2(\bmod 8))$ the pseudoautomorphism $\mathcal{A} \rightarrow \overline{\mathcal{A}}$ (charge conjugation $C$ ) is reduced to the identical transformation Id, therefore, such algebras correspond to truly neutral particles (for example, photons, $K^{0}$-mesons and so on).

The algebra $C \ell_{3,2}$ admits a decomposition $C \ell_{3,2} \simeq C \ell_{3,2}^{+} \oplus C \ell_{3,2}^{+}$, where $C \ell_{3,2}^{+} \simeq C \ell_{2,2}$ is an even subalgebra of $C_{3,2}$. In turn, this decomposition can be represented by the scheme

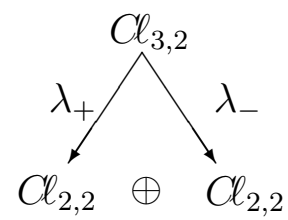

Here central idempotents

$$
\lambda_{+}=\frac{1+\mathbf{e}_{1} \mathbf{e}_{2} \mathbf{e}_{3} \mathbf{e}_{4} \mathbf{e}_{5}}{2}, \quad \lambda_{-}=\frac{1-\mathbf{e}_{1} \mathbf{e}_{2} \mathbf{e}_{3} \mathbf{e}_{4} \mathbf{e}_{5}}{2}
$$

generate two mutually annihilating simple ideals.

Further, the decomposition $C \ell_{3,2} \simeq C_{2,2} \oplus C \ell_{2,2}$ induces a left-regular spinor representation $C \ell_{3,2} \stackrel{\gamma}{\longrightarrow} \operatorname{End}_{\mathbb{R} \oplus \hat{\mathbb{R}}}\left(\mathbb{S}_{4} \oplus \hat{\mathbb{S}}_{4}\right)$, where $\mathbb{S}_{4}(\mathbb{R}) \simeq I_{2,2}=C \ell_{2,2} f$ is a minimal left ideal of the subalgebra $C \ell_{2,2}, f$ is a primitive idempotent of $C \ell_{2,2}$. In general, this spinor representation is realized within the matrix algebra ${ }^{2} \operatorname{Mat}_{4}(\mathbb{R})$.

There is also a homomorphic mapping

$$
\epsilon: \mathrm{Cl}_{3,2} \longrightarrow{ }^{\epsilon} \mathrm{Cl}_{2,2} \text {, }
$$

where

$$
{ }^{\epsilon} \mathrm{Cl}_{2,2} \simeq \mathrm{Cl}_{2,2} / \mathrm{Ker} \epsilon
$$

is a quotient algebra. In accordance with Theorem 14 in [28] at the mapping $\epsilon$ : $\mathrm{Cl}_{3,2} \rightarrow{ }^{\epsilon} \mathrm{Cl}_{2,2}$ we can transfer onto ${ }^{\epsilon} \mathrm{Cl}_{2,2}$ only the antiautomorphism $\mathcal{A} \rightarrow \widetilde{\mathcal{A}}$ (time reversal $T)$. Therefore, we have $\mathrm{PT}\left({ }^{\epsilon} C \ell_{2,2}\right) \simeq\{1, T\} \simeq\{\mathrm{I}, \mathrm{E}\}$. It is easy to see that $\mathrm{PT}\left({ }^{\epsilon} C \ell_{2,2}\right) \simeq\{1, T\}$ is the subgroup of $\mathrm{PT}-\left(C \ell_{2,2}\right) \simeq \mathbb{Z}_{4}$ (see (A.9)) considered in the subsection 3.5. In the spinbasis (A.8), defined by the idempotent $f_{1}^{+} \in C_{2,2}$, we obtain

$$
\mathrm{PT}-\left({ }^{\epsilon} C \ell_{2,2}\right) \simeq\{1, T\} \simeq\left\{\mathbf{1}_{4}, \gamma_{34}\right\} \simeq \mathbb{Z}_{4} / \mathbb{Z}_{2} .
$$

The multiplication table of $\mathrm{PT}_{-}\left({ }^{\epsilon} C \ell_{2,2}\right)$ is given in the Tab. 7 . The group $\mathbb{Z}_{4} / \mathbb{Z}_{2} \simeq$ $\{1, i\}$ has element $i$ with order 4 .

In conclusion it should be noted that anti-de Sitter space $\mathbb{R}^{2,3}$ with the opposite signature $(+,+,-,-,-)$ corresponds to the algebra $C_{2,3}$ of the type $p-q \equiv 7$ $(\bmod 8)$. This algebra has the complex division ring $\mathbb{K} \simeq \mathbb{C}$ and, therefore, there is an isomorphism $C \ell_{2,3} \simeq C \ell_{4}$. It is obvious that in this case we return to CPT groups considered in the section 3 . 


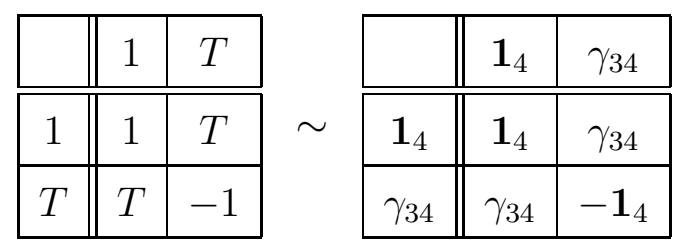

Tab. 7: The multiplication table of PT- $\left({ }^{\epsilon} C_{2,2}\right) \simeq \mathbb{Z}_{4} / \mathbb{Z}_{2}$.

\section{Summary}

In this paper we have study discrete symmetries for the spinor field in the spaces $\mathbb{R}^{4,1}, \mathbb{R}^{1,4}$ and $\mathbb{R}^{3,2}$ in terms of automorphism groups of the algebras $C \ell_{4,1}, C \ell_{1,4}$ and $C_{3,2}$. The algebras $C \ell_{4,1}, C \ell_{1,4}$ and $C \ell_{3,2}$ are considered here as three model examples of the more general algebraic framework. As is known, the spinor field (the field of the spin-1/2) is described within $(1 / 2,0) \oplus(0,1 / 2)$-representation of the Lorentz group. This representation is a fundamental representation of the group $\operatorname{Spin}_{+}(1,3)$. For that reason Dirac wrote that spin- $1 / 2$ is more elementary rather then spin-0 62. All other (finite-dimensional) representations of $\operatorname{Spin}_{+}(1,3)$, which compound higher spin fields, are derived by tensoring of spin-1/2 fields. There is a relationship between representations of $\mathbf{S p i n}_{+}(1,3)$ and Clifford algebras [32, 60]. At this point, complex representations of $\mathbf{S p i n}_{+}(1,3)$ are described within a representation system $\mathfrak{M}_{\mathbb{C}}=\mathfrak{M}^{0} \oplus \mathfrak{M}^{1}$, and real representations within a system $\mathfrak{M}_{\mathbb{R}}=\mathfrak{M}^{+} \oplus \mathfrak{M}^{-}$, where

1) $\mathbb{F}=\mathbb{C}, \mathfrak{M}_{\mathbb{C}}=\mathfrak{M}^{0} \oplus \mathfrak{M}^{1}$.

$$
\begin{array}{lll}
\mathfrak{M}^{0} & : & \mathfrak{C}^{l_{0}+l_{1}-1, l_{0}-l_{1}+1}, \leftrightarrow C \ell_{n}, n \equiv 0 \quad(\bmod 2) \\
\mathfrak{M}^{1}: & { }^{\epsilon} \mathfrak{C}^{l_{0}+l_{1}-1, l_{0}-l_{1}+1}, \leftrightarrow C \ell_{n}, n \equiv 1 \quad(\bmod 2) .
\end{array}
$$

2) $\mathbb{F}=\mathbb{R}, \mathfrak{M}_{\mathbb{R}}=\mathfrak{M}^{+} \oplus \mathfrak{M}^{-}$.

$$
\begin{gathered}
\mathfrak{M}^{+}:\left\{\begin{aligned}
\mathfrak{R}_{0}^{l_{0}} & \leftrightarrow C \ell_{p, q}, p-q \equiv 0 \quad(\bmod 8), \mathbb{K} \simeq \mathbb{R} ; \\
\mathfrak{R}_{2}^{l_{0}} & \leftrightarrow C \ell_{p, q}, p-q \equiv 2 \quad(\bmod 8), \mathbb{K} \simeq \mathbb{R} ; \\
\mathfrak{H}_{4}^{l_{0}} & \leftrightarrow C \ell_{p, q}, p-q \equiv 4 \quad(\bmod 8), \mathbb{K} \simeq \mathbb{H} ; \\
\mathfrak{H}_{6}^{l_{0}} & \leftrightarrow C \ell_{p, q}, p-q \equiv 6 \quad(\bmod 8), \mathbb{K} \simeq \mathbb{H} .
\end{aligned}\right. \\
\mathfrak{M}^{-}:\left\{\begin{array}{ccc}
\mathfrak{C}_{3}^{l_{0}} & \leftrightarrow \quad C \ell_{p, q}, p-q \equiv 3 \quad(\bmod 8), \mathbb{K} \simeq \mathbb{C} ; \\
\mathfrak{C}_{7}^{l_{0}} & \leftrightarrow \quad C \ell_{p, q}, p-q \equiv 7 \quad(\bmod 8), \mathbb{K} \simeq \mathbb{C} ; \\
\mathfrak{R}_{0,2}^{l_{0}} \oplus \mathfrak{R}_{0,2}^{l_{0}} & \leftrightarrow \quad C \ell_{p, q}, p-q \equiv 1 \quad(\bmod 8), \mathbb{K} \simeq \mathbb{R} \oplus \mathbb{R} ; \\
\mathfrak{H}_{4,6}^{l_{0}} \oplus \mathfrak{H}_{4,6}^{l_{0}} & \leftrightarrow \quad C \ell_{p, q}, p-q \equiv 5 \quad(\bmod 8), \mathbb{K} \simeq \mathbb{H} \oplus \mathbb{H} .
\end{array}\right.
\end{gathered}
$$

Here the numbers $l_{0}$ and $l_{1}$ define a Gel'fand-Naimark representation basis of the Lorentz group (for more details see [32, 60]). Hence it follows that the system $\mathfrak{M}_{\mathbb{C}}=\mathfrak{M}^{0} \oplus \mathfrak{M}^{1}$ and also the representations $\mathfrak{C}_{3}^{l_{0}}$ and $\mathfrak{C}_{7}^{l_{0}}$ of the block $\mathfrak{M}^{-}$correspond 
to charged particles. The representations $\mathfrak{H}_{4}^{l_{0}}$ and $\mathfrak{H}_{6}^{l_{0}}$ of the block $\mathfrak{M}^{+}$and $\mathfrak{H}_{4,6}^{l_{0}} \oplus \mathfrak{H}_{4,6}^{l_{0}}$ of $\mathfrak{M}^{-}$(quaternionic representations of the group $\operatorname{Spin}_{+}(1,3)$ ) correspond to neutral particles of the first class (neutral particles which admit particle-antiparticle interchange). In turn, the representations $\mathfrak{R}_{0}^{l_{0}}$ and $\mathfrak{R}_{2}^{l_{0}}$ of the block $\mathfrak{M}^{+}$and $\mathfrak{R}_{0,2}^{l_{0}} \oplus \mathfrak{R}_{0,2}^{l_{0}}$ of $\mathfrak{M}^{-}$correspond to truly neutral particles.

\section{Appendix A: $C P T$ groups of the Dirac algebra $C_{4}$}

In this appendix we will define $C P T$ groups for the algebra $C \ell_{4,1} \simeq C \ell_{4}$ in dependence on the division ring structure of the real subalgebras $C \ell_{p, q} \subset C \ell_{4}$. We consider in sequence $C P T$ groups generated by the subalgebras $C_{1,3}, C \ell_{4,0}, C \ell_{0,4}, C \ell_{3,1}, C \ell_{2,2}$.

\section{A.1 The subalgebra $C_{1,3}$}

The primitive idempotent of the spacetime algebra $C \ell_{1,3}$ can be defined by an expression $f=\frac{1}{2}\left(1+\mathbf{e}_{14}\right)$, and a division ring has the form ${ }^{3}$

$$
\mathbb{K}=f C \ell_{1,3} f=\left\{1, \mathbf{e}_{2}, \mathbf{e}_{3}, \mathbf{e}_{23}\right\} \simeq\{1, \mathbf{i}, \mathbf{j}, \mathbf{k}\} \simeq \mathbb{H},
$$

where $\mathbf{i}, \mathbf{j}, \mathbf{k}$ are well known quaternion units. Therefore, the mapping $C \ell_{1,3} \stackrel{\gamma}{\longrightarrow}$ $\operatorname{End}_{\mathbb{H}}\left(\mathbb{S}_{2}\right)$ leads to the following representation:

$$
\gamma_{1}=\left[\begin{array}{cc}
0 & \mathbf{1}_{2} \\
\mathbf{1}_{2} & 0
\end{array}\right], \quad \gamma_{2}=\left[\begin{array}{cc}
\mathbf{e}_{2} & 0 \\
0 & -\mathbf{e}_{2}
\end{array}\right], \quad \gamma_{3}=\left[\begin{array}{cc}
\mathbf{e}_{3} & 0 \\
0 & -\mathbf{e}_{3}
\end{array}\right], \quad \gamma_{4}=\left[\begin{array}{cc}
0 & -\mathbf{1}_{2} \\
\mathbf{1}_{2} & 0
\end{array}\right] .
$$

Let us define $C P T$ group for the algebra $C \ell_{1,3}$. First of all, $C \ell_{1,3}$ has the type $p-q \equiv 6(\bmod 8)$, therefore, all the eight automorphisms exist (see Theorem 10 in [22]). Using $\gamma$-matrices of the spinbasis (A.1), we define elements of the group $\mathrm{CPT}\left(C \ell_{1,3}\right)$. The matrix of the automorphism $\mathcal{A} \rightarrow \mathcal{A}^{\star}$ has the form $\mathrm{W}=\gamma_{1} \gamma_{2} \gamma_{3} \gamma_{4}=$ $\gamma_{1234}$. Further, since

$$
\gamma_{1}^{t}=\gamma_{1}, \quad \gamma_{2}^{t}=\gamma_{2}, \quad \gamma_{3}^{t}=\gamma_{3}, \quad \gamma_{4}^{t}=-\gamma_{4},
$$

then in accordance with $\widetilde{\mathrm{A}}=\mathrm{EA}^{t} \mathrm{E}^{-1}$ we have

$$
\gamma_{1}=\mathrm{E} \gamma_{1} \mathrm{E}^{-1}, \quad \gamma_{2}=\mathrm{E} \gamma_{2} \mathrm{E}^{-1}, \quad \gamma_{3}=\mathrm{E} \gamma_{3} \mathrm{E}^{-1}, \quad \gamma_{4}=-\mathrm{E} \gamma_{4} \mathrm{E}^{-1} .
$$

Hence it follows that $\mathrm{E}$ commutes with $\gamma_{1}, \gamma_{2}, \gamma_{3}$ and anticommutes with $\gamma_{4}$, that is, $\mathrm{E}=\gamma_{123}$. From the definition $\mathrm{C}=\mathrm{EW}$ we find that the matrix of the antiautomorphism $\mathcal{A} \rightarrow \widetilde{\mathcal{A}^{\star}}$ has the form $\mathrm{C}=\gamma_{4}$. The spinbasis (A.1) contains both complex and real matrices:

$$
\gamma_{1}^{*}=\gamma_{1}, \quad \gamma_{2}^{*}=-\gamma_{2}, \quad \gamma_{3}^{*}=-\gamma_{3}, \quad \gamma_{4}^{*}=\gamma_{4},
$$

\footnotetext{
${ }^{3}$ Here we use the CLIFFORD package for Maple developed by R. Abłamowicz [50].
} 
choosing $\mathbf{e}_{2} \sim \mathbf{i} \sim\left[\begin{array}{cc}0 & -i \\ i & 0\end{array}\right], \mathbf{e}_{3} \sim \mathbf{j} \sim\left[\begin{array}{cc}i & 0 \\ 0 & -i\end{array}\right]$. Therefore, from $\overline{\mathrm{A}}=\Pi \mathrm{A}^{*} \Pi^{-1}$ we obtain

$$
\gamma_{1}=\Pi \gamma_{1} \Pi^{-1}, \quad \gamma_{2}=-\Pi \gamma_{2} \Pi^{-1}, \quad \gamma_{3}=-\Pi \gamma_{3} \Pi^{-1}, \quad \gamma_{4}=\Pi \gamma_{4} \Pi^{-1}
$$

From the latter relations we find $\Pi=\gamma_{23}$. Further, in accordance with $\mathrm{K}=\Pi \mathrm{W}$ for the spinor representation of the pseudoautomorphism $\mathcal{A} \rightarrow \overline{\mathcal{A}^{\star}}$ we have $\mathrm{K}=\gamma_{14}$. Finally, for the pseudoantiautomorphisms $\mathcal{A} \rightarrow \overline{\widetilde{\mathcal{A}}}\left(C T\right.$-transformation) and $\mathcal{A} \rightarrow \overline{\widetilde{\mathcal{A}}^{\star}}$ (CPT-transformation) from the definitions $\mathbf{S}=\Pi \mathrm{E}$ and $\mathrm{F}=\Pi \mathrm{C}$ we find that $\mathrm{S}=\gamma_{1}$ and $\mathrm{F}=\gamma_{234}$. Thus, we come to the following automorphism group:

$$
\begin{aligned}
\operatorname{CPT}\left(C \ell_{1,3}\right) \simeq\{\mathrm{I}, \mathrm{W}, \mathrm{E}, \mathrm{C}, \Pi, \mathrm{K}, \mathrm{S}, \mathrm{F}\} \simeq & \\
& \simeq\left\{\mathbf{1}_{4}, \gamma_{1234}, \gamma_{123}, \gamma_{4}, \gamma_{23}, \gamma_{14}, \gamma_{1}, \gamma_{234}\right\}
\end{aligned}
$$

The multiplication table of this group is given in the Tab. 8. Super and subscripts in $\mathrm{CPT}_{i}^{+}$correspond to super and subscripts of the primitive idempotents $f_{i}^{+}$. As

\begin{tabular}{|c||c|c|c|c|c|c|c|c|}
\hline & $\mathbf{1}_{4}$ & $\gamma_{1234}$ & $\gamma_{123}$ & $\gamma_{4}$ & $\gamma_{23}$ & $\gamma_{14}$ & $\gamma_{1}$ & $\gamma_{234}$ \\
\hline \hline $\mathbf{1}_{4}$ & $\mathbf{1}_{4}$ & $\gamma_{1234}$ & $\gamma_{123}$ & $\gamma_{4}$ & $\gamma_{23}$ & $\gamma_{14}$ & $\gamma_{1}$ & $\gamma_{234}$ \\
\hline$\gamma_{1234}$ & $\gamma_{1234}$ & $-\mathbf{1}_{4}$ & $\gamma_{4}$ & $-\gamma_{123}$ & $-\gamma_{14}$ & $\gamma_{23}$ & $-\gamma_{234}$ & $\gamma_{1}$ \\
\hline$\gamma_{123}$ & $\gamma_{123}$ & $-\gamma_{4}$ & $-\mathbf{1}_{4}$ & $\gamma_{1234}$ & $-\gamma_{1}$ & $\gamma_{234}$ & $\gamma_{23}$ & $-\gamma_{14}$ \\
\hline$\gamma_{4}$ & $\gamma_{4}$ & $\gamma_{123}$ & $-\gamma_{1234}$ & $-\mathbf{1}_{4}$ & $\gamma_{234}$ & $\gamma_{1}$ & $-\gamma_{14}$ & $-\gamma_{23}$ \\
\hline$\gamma_{23}$ & $\gamma_{23}$ & $-\gamma_{14}$ & $-\gamma_{1}$ & $\gamma_{234}$ & $-\mathbf{1}_{4}$ & $\gamma_{1234}$ & $\gamma_{123}$ & $-\gamma_{4}$ \\
\hline$\gamma_{14}$ & $\gamma_{14}$ & $\gamma_{23}$ & $-\gamma_{234}$ & $-\gamma_{1}$ & $\gamma_{1234}$ & $\mathbf{1}_{4}$ & $-\gamma_{4}$ & $-\gamma_{123}$ \\
\hline$\gamma_{1}$ & $\gamma_{1}$ & $\gamma_{234}$ & $\gamma_{23}$ & $\gamma_{14}$ & $\gamma_{123}$ & $\gamma_{4}$ & $\mathbf{1}_{4}$ & $\gamma_{1234}$ \\
\hline$\gamma_{234}$ & $\gamma_{234}$ & $-\gamma_{1}$ & $\gamma_{14}$ & $-\gamma_{23}$ & $-\gamma_{4}$ & $\gamma_{123}$ & $-\gamma_{1234}$ & $\mathbf{1}_{4}$ \\
\hline
\end{tabular}

Tab. 8: The multiplication table of $\mathrm{CPT}_{1}^{+}\left(C \ell_{1,3}\right)$.

follows from this table the group $\mathrm{CPT}_{1}^{+}\left(C_{1,3}\right)$ is an non-Abelian finite group with the signature $(-,-,-,-,+,+,+)$. The $C P T$ group $C^{-,-,-,-,+,+,+} \simeq \stackrel{*}{\mathbb{Z}}_{4} \times \mathbb{Z}_{2} \times \mathbb{Z}_{2}$ is the subgroup of $G(4,1)$. It is easy to verify that the same group we obtain for the idempotent $f=\frac{1}{2}\left(1-\mathbf{e}_{14}\right)$.

In common with the idempotents $f_{1}^{ \pm}=\frac{1}{2}\left(1 \pm \mathbf{e}_{14}\right)$ the algebra $C \ell_{1,3}$ has the following sequence of primitive idempotents:

$$
f_{2}^{ \pm}=\frac{1}{2}\left(1 \pm \mathbf{e}_{1}\right), \quad f_{3}^{ \pm}=\frac{1}{2}\left(1 \pm \mathbf{e}_{12}\right), \quad f_{4}^{ \pm}=\frac{1}{2}\left(1 \pm \mathbf{e}_{13}\right), \quad f_{5}^{ \pm}=\frac{1}{2}\left(1 \pm \mathbf{e}_{234}\right) .
$$


It is easy to verify that idempotents $f_{2}^{+}, f_{3}^{+}, f_{4}^{+}$lead with minor variations to the group $\stackrel{*}{\mathbb{Z}}_{4} \times \mathbb{Z}_{2} \times \mathbb{Z}_{2}$. We list below division rings, spinbases and $C P T$ groups for $f_{2}^{+}$, $f_{3}^{+}, f_{4}^{+}$:

$$
\begin{aligned}
& f_{2}^{+}=\frac{1}{2}\left(1+\mathbf{e}_{1}\right) \\
& \mathbb{K}=f_{2}^{+} C \ell_{1,3} f_{2}^{+}=\left\{1, \mathbf{e}_{23}, \mathbf{e}_{24}, \mathbf{e}_{34}\right\} \simeq\{1, \mathbf{i}, \mathbf{j}, \mathbf{k}\} \simeq \mathbb{H}, \\
& \gamma_{1}=\left[\begin{array}{cc}
\mathbf{1}_{2} & 0 \\
0 & -\mathbf{1}_{2}
\end{array}\right], \gamma_{2}=\left[\begin{array}{cc}
0 & -\mathbf{1}_{2} \\
\mathbf{1}_{2} & 0
\end{array}\right], \gamma_{3}=\left[\begin{array}{cc}
0 & \mathbf{e}_{23} \\
\mathbf{e}_{23} & 0
\end{array}\right], \gamma_{4}=\left[\begin{array}{cc}
0 & \mathbf{e}_{24} \\
\mathbf{e}_{24} & 0
\end{array}\right] \text {, } \\
& \mathrm{CPT}_{2}^{+}\left(C \ell_{1,3}\right) \simeq\left\{\mathbf{1}_{4}, \gamma_{1234}, \gamma_{134}, \gamma_{2}, \gamma_{34}, \gamma_{12}, \gamma_{1}, \gamma_{234}\right\} \simeq \stackrel{*}{\mathbb{Z}}_{4} \times \mathbb{Z}_{2}, \\
& C^{-,-,-,-,+,+,+} \simeq \stackrel{*}{\mathbb{Z}}_{4} \times \mathbb{Z}_{2} \times \mathbb{Z}_{2} \\
& f_{3}^{+}=\frac{1}{2}\left(1+\mathbf{e}_{12}\right) \\
& \mathbb{K}=f_{3}^{+} C \ell_{1,3} f_{3}^{+}=\left\{1, \mathbf{e}_{3}, \mathbf{e}_{4}, \mathbf{e}_{34}\right\} \simeq\{1, \mathbf{i}, \mathbf{j}, \mathbf{k}\} \simeq \mathbb{H}, \\
& \gamma_{1}=\left[\begin{array}{cc}
0 & \mathbf{1}_{2} \\
\mathbf{1}_{2} & 0
\end{array}\right], \quad \gamma_{2}=\left[\begin{array}{cc}
0 & -\mathbf{1}_{2} \\
\mathbf{1}_{2} & 0
\end{array}\right], \quad \gamma_{3}=\left[\begin{array}{cc}
\mathbf{e}_{3} & 0 \\
0 & -\mathbf{e}_{3}
\end{array}\right], \quad \gamma_{4}=\left[\begin{array}{cc}
\mathbf{e}_{4} & 0 \\
0 & -\mathbf{e}_{4}
\end{array}\right] \text {, } \\
& \mathrm{CPT}_{3}^{+}\left(C \ell_{1,3}\right) \simeq\left\{\mathbf{1}_{4}, \gamma_{1234}, \gamma_{134}, \gamma_{2}, \gamma_{34}, \gamma_{12}, \gamma_{2}, \gamma_{234}\right\} \simeq \stackrel{*}{\mathbb{Z}}_{4} \times \mathbb{Z}_{2} \text {, } \\
& C^{-,-,-,-,+,+,+} \simeq \stackrel{*}{\mathbb{Z}}_{4} \times \mathbb{Z}_{2} \times \mathbb{Z}_{2} \\
& f_{4}^{+}=\frac{1}{2}\left(1+\mathbf{e}_{13}\right) \\
& \mathbb{K}=f_{4}^{+} C \ell_{1,3} f_{4}^{+}=\left\{1, \mathbf{e}_{2}, \mathbf{e}_{4}, \mathbf{e}_{24}\right\} \simeq\{1, \mathbf{i}, \mathbf{j}, \mathbf{k}\} \simeq \mathbb{H}, \\
& \gamma_{1}=\left[\begin{array}{cc}
0 & \mathbf{1}_{2} \\
\mathbf{1}_{2} & 0
\end{array}\right], \quad \gamma_{2}=\left[\begin{array}{cc}
\mathbf{e}_{2} & 0 \\
0 & -\mathbf{e}_{2}
\end{array}\right], \quad \gamma_{3}=\left[\begin{array}{cc}
0 & -\mathbf{1}_{2} \\
\mathbf{1}_{2} & 0
\end{array}\right], \quad \gamma_{4}=\left[\begin{array}{cc}
\mathbf{e}_{4} & 0 \\
0 & -\mathbf{e}_{4}
\end{array}\right] \text {, } \\
& \mathrm{CPT}_{4}^{+}\left(C \ell_{1,3}\right) \simeq\left\{\mathbf{1}_{4}, \gamma_{1234}, \gamma_{124}, \gamma_{3}, \gamma_{24}, \gamma_{13}, \gamma_{1}, \gamma_{234}\right\} \simeq \mathbb{Z}_{4}^{*} \times \mathbb{Z}_{2}, \\
& C^{-,-,-,-,+,+,+} \simeq \stackrel{*}{\mathbb{Z}}_{4} \times \mathbb{Z}_{2} \times \mathbb{Z}_{2} \text {. }
\end{aligned}
$$

It is not hard to see that idempotents $f_{1}^{-} f_{2}^{-}, f_{3}^{-}, f_{4}^{-}$lead to the same group $\stackrel{*}{\mathbb{Z}}_{4} \times$ $\mathbb{Z}_{2} \times \mathbb{Z}_{2}$.

In contrast to $f_{1}^{ \pm} f_{2}^{ \pm}, f_{3}^{ \pm}, f_{4}^{ \pm}$, for the primitive idempotents $f_{5}^{ \pm}=\frac{1}{2}\left(1 \pm \mathbf{e}_{234}\right)$ we have other realizations of $C^{a, b, c, d, e, f, g}$. Indeed,

$$
\begin{gathered}
f_{5}^{+}=\frac{1}{2}\left(1+\mathbf{e}_{234}\right), \\
\mathbb{K}=f_{5}^{+} C \ell_{1,3} f_{5}^{+}=\left\{1, \mathbf{e}_{2}, \mathbf{e}_{3}, \mathbf{e}_{23}\right\} \simeq\{1, \mathbf{i}, \mathbf{j}, \mathbf{k}\} \simeq \mathbb{H}, \\
\gamma_{1}=\left[\begin{array}{cc}
0 & \mathbf{1}_{2} \\
\mathbf{1}_{2} & 0
\end{array}\right], \quad \gamma_{2}=\left[\begin{array}{cc}
\mathbf{e}_{2} & 0 \\
0 & -\mathbf{e}_{2}
\end{array}\right], \quad \gamma_{3}=\left[\begin{array}{cc}
\mathbf{e}_{3} & 0 \\
0 & -\mathbf{e}_{3}
\end{array}\right], \quad \gamma_{4}=\left[\begin{array}{cc}
-\mathbf{e}_{23} & 0 \\
0 & \mathbf{e}_{23}
\end{array}\right] .
\end{gathered}
$$


In the spinbasis (A.2) we see that all the $\gamma$-matrices are symmetric,

$$
\gamma_{1}^{t}=\gamma_{1}, \quad \gamma_{2}^{t}=\gamma_{2}, \quad \gamma_{3}^{t}=\gamma_{3}, \quad \gamma_{4}^{t}=\gamma_{4}
$$

Therefore, the matrix $\mathrm{E}$ of the antiautomorphism $\mathcal{A} \rightarrow \widetilde{\mathcal{A}}$ commutes with all the matrices of (A.2). This condition takes place only in the case when $E \sim \mathbf{1}_{4}$. We suppose $\mathrm{E}=\eta_{t} \mathbf{1}_{4}$, where $\eta_{t}$ is a phase factor, $\eta_{t} \in \mathbb{C}^{*}=\mathbb{C}-\{0\}$. Then from the definition $\mathrm{C}=\mathrm{EW}$ we have $\mathrm{C}=\eta_{t} \gamma_{1234}$ for the antiautomorphism $\mathcal{A} \rightarrow \widetilde{\mathcal{A}^{\star}}$. The basis (A.2) contains both complex and real matrices:

$$
\gamma_{1}^{*}=\gamma_{1}, \quad \gamma_{2}^{*}=-\gamma_{2}, \quad \gamma_{3}^{*}=-\gamma_{3}, \quad \gamma_{4}^{*}=-\gamma_{4} \text {. }
$$

Hence it follows that the matrix $\Pi$ of the pseudoautomorphism $\mathcal{A} \rightarrow \overline{\mathcal{A}}$ commutes with $\gamma_{1}$ and anticommutes with $\gamma_{2}, \gamma_{3}, \gamma_{4}$. Therefore, the spinor representation of $\mathcal{A} \rightarrow \overline{\mathcal{A}}$ is defined by $\Pi=\gamma_{1}$. Further, from the definition $\mathrm{K}=\Pi \mathrm{W}$ we have $\mathrm{K}=\gamma_{234}$ for the pseudoautomorphism $\mathcal{A} \rightarrow \overline{\mathcal{A}^{\star}}$. Finally, from the definitions $\mathrm{S}=\Pi \mathrm{E}$ and $\mathrm{F}=\Pi \mathrm{C}$ we obtain for the pseudoantiautomorphisms $\mathcal{A} \rightarrow \overline{\widetilde{\mathcal{A}}}$ and $\mathcal{A} \rightarrow \overline{\widetilde{\mathcal{A}^{\star}}}$ the following spinor representations: $\mathrm{S}=\eta_{t} \gamma_{1}$ and $\mathrm{F}=\eta_{t} \gamma_{234}$. Thus, we come to the following generating group:

$$
\mathrm{CPT}_{5}^{+}\left(C \ell_{1,3}\right) \simeq\left\{\mathbf{1}_{4}, \gamma_{1234}, \eta_{t} \mathbf{1}_{4}, \eta_{t} \gamma_{1234}, \gamma_{1}, \gamma_{234}, \eta_{t} \gamma_{1}, \eta_{t} \gamma_{234}\right\}
$$

The multiplication table of this group is shown in the Tab.9. As follows from this

\begin{tabular}{|c||c|c|c|c|c|c|c|c|}
\hline & $\mathbf{1}_{4}$ & $\boldsymbol{\omega}$ & $\eta_{t} \mathbf{1}_{4}$ & $\eta_{t} \boldsymbol{\omega}$ & $\gamma_{1}$ & $\gamma_{234}$ & $\eta_{t} \gamma_{1}$ & $\eta_{t} \gamma_{234}$ \\
\hline \hline $\mathbf{1}_{4}$ & $\mathbf{1}_{4}$ & $\boldsymbol{\omega}$ & $\eta_{t} \mathbf{1}_{4}$ & $\eta_{t} \boldsymbol{\omega}$ & $\gamma_{1}$ & $\gamma_{234}$ & $\eta_{2} \gamma_{1}$ & $\eta_{t} \gamma_{234}$ \\
\hline $\boldsymbol{\omega}$ & $\boldsymbol{\omega}$ & $-\mathbf{1}_{4}$ & $\eta_{t} \boldsymbol{\omega}$ & $-\eta_{t} \mathbf{1}_{4}$ & $-\gamma_{234}$ & $\gamma_{1}$ & $-\eta_{t} \gamma_{234}$ & $\eta_{t} \gamma_{1}$ \\
\hline$\eta_{t} \mathbf{1}_{4}$ & $\eta_{t} \mathbf{1}_{4}$ & $\eta_{t} \boldsymbol{\omega}$ & $\eta_{t}^{2} \mathbf{1}_{4}$ & $\eta_{t}^{2} \boldsymbol{\omega}$ & $\eta_{t} \gamma_{1}$ & $\eta_{t} \gamma_{234}$ & $\eta_{t}^{2} \gamma_{1}$ & $\eta_{t}^{2} \gamma_{234}$ \\
\hline$\eta_{t} \boldsymbol{\omega}$ & $\eta_{t} \boldsymbol{\omega}$ & $-\eta_{t} \mathbf{1}_{4}$ & $\eta_{t}^{2} \boldsymbol{\omega}$ & $-\eta_{t}^{2} \mathbf{1}_{4}$ & $-\eta_{t} \gamma_{234}$ & $\eta_{t} \gamma_{1}$ & $-\eta_{t}^{2} \gamma_{234}$ & $\eta_{t}^{2} \gamma_{1}$ \\
\hline$\gamma_{1}$ & $\gamma_{1}$ & $\gamma_{234}$ & $\eta_{t} \gamma_{1}$ & $\eta_{t} \gamma_{234}$ & $\mathbf{1}_{4}$ & $\boldsymbol{\omega}$ & $\eta_{t} \mathbf{1}_{4}$ & $\eta_{t} \boldsymbol{\omega}$ \\
\hline$\gamma_{234}$ & $\gamma_{234}$ & $-\gamma_{1}$ & $\eta_{t} \gamma_{234}$ & $-\eta_{t} \gamma_{1}$ & $-\boldsymbol{\omega}$ & $\mathbf{1}_{4}$ & $-\eta_{t} \boldsymbol{\omega}$ & $\eta_{t} \mathbf{1}_{4}$ \\
\hline$\eta_{t} \gamma_{1}$ & $\eta_{t} \gamma_{1}$ & $\eta_{t} \gamma_{234}$ & $\eta_{t}^{2} \gamma_{1}$ & $\eta_{t}^{2} \gamma_{234}$ & $\eta_{t} \mathbf{1}_{4}$ & $\eta_{t} \boldsymbol{\omega}$ & $\eta_{t}^{2} \mathbf{1}_{4}$ & $\eta_{t}^{2} \boldsymbol{\omega}$ \\
\hline$\eta_{t} \gamma_{234}$ & $\eta_{t} \gamma_{234}$ & $-\eta_{t} \gamma_{1}$ & $\eta_{t}^{2} \gamma_{234}$ & $-\eta_{t}^{2} \gamma_{1}$ & $-\eta_{t} \boldsymbol{\omega}$ & $\eta_{t} \mathbf{1}_{4}$ & $-\eta_{t}^{2} \boldsymbol{\omega}$ & $\eta_{t}^{2} \mathbf{1}_{4}$ \\
\hline
\end{tabular}

Tab. 9: The multiplication table of $\mathrm{CPT}_{5}^{+}\left(C \ell_{1,3}\right)$ in dependence of the sign of $\eta_{t}^{2}$, where $\boldsymbol{\omega}=\gamma_{1234}$. $\mathrm{CPT}_{5}^{+}\left(C_{1,3}\right) \simeq D_{4}$ at $\eta_{t}^{2}=1$ and $\mathrm{CPT}_{5}^{+}\left(C \ell_{1,3}\right) \simeq$ $\stackrel{*}{\mathbb{Z}}_{4} \times \mathbb{Z}_{2}$ at $\eta_{t}^{2}=-1$.

table, when $\eta_{t}^{2}=1$ we have $C^{-,+,-,+,+,+,+} \simeq D_{4} \times \mathbb{Z}_{2}$ and $C^{-,-,+,+,+,-,-} \simeq \stackrel{*}{\mathbb{Z}}_{4} \times \mathbb{Z}_{2} \times \mathbb{Z}_{2}$ when $\eta_{t}^{2}=-1$. In the first case $\eta_{t}= \pm 1$, and in the second case $\eta_{t}= \pm i$. 


\section{A.2 The subalgebra $C_{4,0}$}

The next real subalgebra of $C \ell_{4}$ is $C \ell_{4,0}$. This algebra has the type $p-q \equiv 4(\bmod 8)$ and the first primitive idempotent $f_{1}^{+}=\frac{1}{2}\left(1+\mathbf{e}_{1}\right)$. The division ring of $C \ell_{4,0}$ is

$$
\mathbb{K}=f_{1}^{+} C \ell_{4,0} f_{1}^{+}=\left\{1, \mathbf{e}_{23}, \mathbf{e}_{24}, \mathbf{e}_{34}\right\} \simeq\{1, \mathbf{i}, \mathbf{j}, \mathbf{k}\} \simeq \mathbb{H}
$$

Therefore, the mapping $C \ell_{4,0} \stackrel{\gamma}{\longrightarrow} \operatorname{End}_{\mathbb{H}}\left(\mathbb{S}_{2}\right)$ gives the following spinor representation:

$$
\gamma_{1}=\left[\begin{array}{cc}
\mathbf{1}_{2} & 0 \\
0 & -\mathbf{1}_{2}
\end{array}\right], \quad \gamma_{2}=\left[\begin{array}{cc}
0 & \mathbf{1}_{2} \\
\mathbf{1}_{2} & 0
\end{array}\right], \quad \gamma_{3}=\left[\begin{array}{cc}
0 & -\mathbf{e}_{23} \\
\mathbf{e}_{23} & 0
\end{array}\right], \quad \gamma_{4}=\left[\begin{array}{cc}
0 & -\mathbf{e}_{24} \\
\mathbf{e}_{24} & 0
\end{array}\right]
$$

Making the same calculations as in the case of ${C \ell_{1,3}}_{1}$ we come to the group

$$
\mathrm{CPT}_{1}^{+}\left(C \ell_{4,0}\right) \simeq\left\{\mathbf{1}_{4}, \gamma_{1234}, \gamma_{34}, \gamma_{12}, \eta_{c} \gamma_{34}, \eta_{c} \gamma_{12}, \eta_{c} \mathbf{1}_{4}, \eta_{c} \gamma_{1234}\right\}
$$

Hence it follows that $\mathrm{CPT}_{1}^{+}\left(C \ell_{4,0}\right) \simeq \stackrel{*}{\mathbb{Z}}_{4} \times \mathbb{Z}_{2}$ at $\eta_{c}^{2}=1$ and also $\mathrm{CPT}_{1}^{+}\left(C \ell_{4,0}\right) \simeq \stackrel{*}{\mathbb{Z}}_{4} \times \mathbb{Z}_{2}$ at $\eta_{c}^{2}=-1$. Therefore, in both cases we have

$$
C^{+,-,-,-,-,+,+} \simeq C^{+,-,-,+,+,-,-} \simeq \mathrm{CPT}_{1}^{+}\left(C \ell_{4,0}\right) \simeq \stackrel{*}{\mathbb{Z}}_{4} \times \mathbb{Z}_{2} \times \mathbb{Z}_{2}
$$

In common with the idempotent $f_{1}^{+}$the algebra $C \ell_{4,0}$ has the following primitive idempotents:

$$
f_{2}^{+}=\frac{1}{2}\left(1+\mathbf{e}_{2}\right), \quad f_{3}^{+}=\frac{1}{2}\left(1+\mathbf{e}_{3}\right), \quad f_{4}^{+}=\frac{1}{2}\left(1+\mathbf{e}_{4}\right), \quad f_{5}^{+}=\frac{1}{2}\left(1+\mathbf{e}_{1234}\right) .
$$

It is easy to verify that the idempotents $f_{2}^{+}, f_{3}^{+}, f_{4}^{+}$lead to the group (A.3) (resp. (A.4) ), that is, $\mathrm{CPT}_{2}^{+}\left(C \ell_{4,0}\right), \mathrm{CPT}_{3}^{+}\left(C \ell_{4,0}\right), \mathrm{CPT}_{4}^{+}\left(C \ell_{4,0}\right)$ are isomorphic to $\mathrm{CPT}_{1}^{+}\left(C \ell_{4,0}\right)$. However, situation is changed for the idempotent $f_{5}^{+}$. In this case we have

$$
\begin{gathered}
\mathbb{K}=f_{5}^{+} C \ell_{4,0} f_{5}^{+}=\left\{1, \mathbf{e}_{23}, \mathbf{e}_{24}, \mathbf{e}_{34}\right\} \simeq\{1, \mathbf{i}, \mathbf{j}, \mathbf{k}\} \simeq \mathbb{H} \\
\gamma_{1}=\left[\begin{array}{cc}
0 & \mathbf{1}_{2} \\
\mathbf{1}_{2} & 0
\end{array}\right], \gamma_{2}=\left[\begin{array}{cc}
0 & \mathbf{e}_{34} \\
-\mathbf{e}_{34} & 0
\end{array}\right], \gamma_{3}=\left[\begin{array}{cc}
0 & -\mathbf{e}_{24} \\
\mathbf{e}_{24} & 0
\end{array}\right], \gamma_{4}=\left[\begin{array}{cc}
0 & \mathbf{e}_{23} \\
-\mathbf{e}_{23} & 0
\end{array}\right] \\
\mathrm{CPT}_{5}^{+}\left(C \ell_{4,0}\right) \simeq\left\{\mathbf{1}_{4}, \gamma_{1234}, \gamma_{1}, \gamma_{234}, \eta_{c} \gamma_{1}, \eta_{c} \gamma_{234}, \eta_{c} \mathbf{1}_{4}, \eta_{c} \gamma_{1234}\right\}
\end{gathered}
$$

and

$$
\begin{gathered}
\eta_{c}^{2}=1: \mathrm{CPT}_{5}^{+}\left(C \ell_{4,0}\right) \simeq D_{4} \longrightarrow C^{+,+,-,+,-,+,+} \simeq D_{4} \times \mathbb{Z}_{2}, \\
\eta_{c}^{2}=-1: \mathrm{CPT}_{5}^{+}\left(C l_{4,0}\right) \simeq \stackrel{\mathbb{Z}}{4}_{4} \times \mathbb{Z}_{2} \longrightarrow C^{+,+,-,-,+,-,} \simeq \stackrel{\mathbb{Z}}{4}_{4} \times \mathbb{Z}_{2} \times \mathbb{Z}_{2} .
\end{gathered}
$$

It is not hard to see that for the idempotents $f_{i}^{-}(i=1, \ldots, 5)$ we obtain the same isomorphisms. 


\section{A.3 The subalgebra $C_{0,4}$}

The next real subalgebra of $C \ell_{4}$ with the quaternionic ring $\mathbb{K}=\mathbb{H}$ is $C \ell_{0,4}$. This algebra has the type $p-q \equiv 4(\bmod 8)$ and the first primitive idempotent $f_{1}^{+}=\frac{1}{2}\left(1+\mathbf{e}_{123}\right)$. The division ring of $C \ell_{0,4}$ for $f_{1}^{+}$is

$$
\mathbb{K}=f_{1}^{+} C \ell_{0,4} f_{1}^{+}=\left\{1, \mathbf{e}_{1}, \mathbf{e}_{13}, \mathbf{e}_{3}\right\} \simeq\{1, \mathbf{i}, \mathbf{j}, \mathbf{k}\} \simeq \mathbb{H} .
$$

Therefore, the mapping $C \ell_{0,4} \stackrel{\gamma}{\longrightarrow} \operatorname{End}_{\mathbb{H}}\left(\mathbb{S}_{2}\right)$ gives the following spinor representation:

$$
\gamma_{1}=\left[\begin{array}{cc}
\mathbf{e}_{1} & 0 \\
0 & -\mathbf{e}_{1}
\end{array}\right], \quad \gamma_{2}=\left[\begin{array}{cc}
\mathbf{e}_{13} & 0 \\
0 & -\mathbf{e}_{13}
\end{array}\right], \quad \gamma_{3}=\left[\begin{array}{cc}
\mathbf{e}_{3} & 0 \\
0 & -\mathbf{e}_{3}
\end{array}\right], \quad \gamma_{4}=\left[\begin{array}{cc}
0 & -\mathbf{1}_{2} \\
\mathbf{1}_{2} & 0
\end{array}\right] \text {. }
$$

The generating group $\mathrm{CPT}_{1}^{+}\left(C_{0,4}\right)$ arising from the spinbasis (A.6) is

$$
\mathrm{CPT}_{1}^{+}\left(C \ell_{0,4}\right) \simeq\left\{\mathbf{1}_{4}, \gamma_{1234}, \gamma_{123}, \gamma_{4}, \eta_{c} \gamma_{4}, \eta_{c} \gamma_{123}, \eta_{c} \gamma_{1234}, \eta_{c} \mathbf{1}_{4}\right\}
$$

In this case we have

$$
\begin{gathered}
\eta_{c}^{2}=1: \mathrm{CPT}_{1}^{+}\left(C \ell_{0,4}\right) \simeq D_{4} \longrightarrow C^{+,+,-,-,+,+,+} \simeq D_{4} \times \mathbb{Z}_{2}, \\
\eta_{c}^{2}=-1: \mathrm{CPT}_{1}^{+}\left(C \ell_{0,4}\right) \simeq \mathbb{Z}_{4}^{*} \times \mathbb{Z}_{2} \longrightarrow C^{+,+,-,+,-,-,-} \simeq \mathbb{Z}_{4}^{*} \times \mathbb{Z}_{2} \times \mathbb{Z}_{2} .
\end{gathered}
$$

It is easy to verify that for other primitive idempotents within $C \ell_{0,4}$,

$$
\begin{aligned}
f_{2}^{ \pm}=\frac{1}{2}\left(1 \pm \mathbf{e}_{124}\right), \quad f_{3}^{ \pm} & =\frac{1}{2}\left(1 \pm \mathbf{e}_{134}\right), \quad f_{4}^{ \pm}=\frac{1}{2}\left(1+ \pm \mathbf{e}_{234}\right), \\
f_{5}^{ \pm} & =\frac{1}{2}\left(1 \pm \mathbf{e}_{1234}\right),
\end{aligned}
$$

all the generating groups $\mathrm{CPT}_{i}^{ \pm}\left(C \ell_{0,4}\right)(i=2, \ldots, 5)$ are isomorphic to $\mathrm{CPT}_{1}^{+}\left(C \ell_{0,4}\right)$.

\section{A.4 The subalgebra $C_{3,1}$}

The first real subalgebra of $C_{4}$ with the real division ring $\mathbb{K}=\mathbb{R}$ is $C \ell_{3,1}$ (so-called Maiorana algebra). This algebra has the type $p-q \equiv 2(\bmod 8)$ and the first primitive idempotent is

$$
f_{1}^{+}=\frac{1}{4}\left(1+\mathbf{e}_{1}\right)\left(1+\mathbf{e}_{34}\right)
$$

and

$$
\mathbb{K}=f_{1}^{+} C \ell_{3,1} f_{1}^{+} \simeq\{1\} \simeq \mathbb{R}
$$

The mapping $C \ell_{3,1} \stackrel{\gamma}{\longrightarrow} \operatorname{End}_{\mathbb{R}}\left(\mathbb{S}_{4}\right)$ gives

$$
\gamma_{1}=\left[\begin{array}{cccc}
1 & 0 & 0 & 0 \\
0 & -1 & 0 & 0 \\
0 & 0 & -1 & 0 \\
0 & 0 & 0 & 1
\end{array}\right], \quad \gamma_{2}=\left[\begin{array}{llll}
0 & 1 & 0 & 0 \\
1 & 0 & 0 & 0 \\
0 & 0 & 0 & 1 \\
0 & 0 & 1 & 0
\end{array}\right]
$$




$$
\gamma_{3}=\left[\begin{array}{cccc}
0 & 0 & 1 & 0 \\
0 & 0 & 0 & -1 \\
1 & 0 & 0 & 0 \\
0 & -1 & 0 & 0
\end{array}\right], \quad \gamma_{4}=\left[\begin{array}{cccc}
0 & 0 & -1 & 0 \\
0 & 0 & 0 & 1 \\
1 & 0 & 0 & 0 \\
0 & -1 & 0 & 0
\end{array}\right]
$$

In case of the type $p-q \equiv 2(\bmod 8)$ the matrix $\Pi$ of the pseudoautomorphism $\mathcal{A} \rightarrow \overline{\mathcal{A}}$ is proportional to the unit matrix (identical transformation) and the automorphism group $\mathrm{CPT}\left(C \ell_{p, q}\right)$ is reduced to the group of fundamental automorphisms, $\mathrm{PT}_{ \pm}\left(C \ell_{p, q}\right)$ (see Theorem 10 in [22]).

For the idempotent $f_{1}^{+}$and the spinbasis (A.7) we have

$$
\begin{gathered}
\mathrm{PT}_{+}\left(C \ell_{3,1}\right) \simeq\left\{\mathbf{1}_{4}, \gamma_{1234}, \gamma_{123}, \gamma_{4}\right\} \simeq Q_{4} / \mathbb{Z}_{2}, \\
C^{-,-,-} \simeq Q_{4} .
\end{gathered}
$$

The idempotent $f_{1}^{-}=\frac{1}{4}\left(1-\mathbf{e}_{1}\right)\left(1-\mathbf{e}_{34}\right)$ leads also to the group $\mathrm{PT}+\left(C \ell_{3,1}\right) \simeq Q_{4} / \mathbb{Z}_{2}$. Moreover, all other primitive idempotents of $C \ell_{3,1}$,

$$
\begin{gathered}
f_{2}^{ \pm}=\frac{1}{4}\left(1 \pm \mathbf{e}_{1}\right)\left(1 \pm \mathbf{e}_{24}\right), \quad f_{3}^{ \pm}=\frac{1}{4}\left(1 \pm \mathbf{e}_{2}\right)\left(1 \pm \mathbf{e}_{14}\right), \\
f_{4}^{ \pm}=\frac{1}{4}\left(1 \pm \mathbf{e}_{3}\right)\left(1 \pm \mathbf{e}_{134}\right), \quad f_{5}^{ \pm}=\frac{1}{4}\left(1 \pm \mathbf{e}_{3} \mathbf{e}_{4}\right)\left(1 \pm \mathbf{e}_{234}\right)
\end{gathered}
$$

generate spinor representations with $\mathrm{PT}_{+}\left(C \ell_{3,1}\right) \simeq Q_{4} / \mathbb{Z}_{2}$. Therefore, the group $C^{-,-,-} \simeq Q_{4}$ is an invariant fact for $C_{3,1}$. In other words, this result does not depend on the choice of the spinor representation.

\section{A.5 The subalgebra $C_{2,2}$}

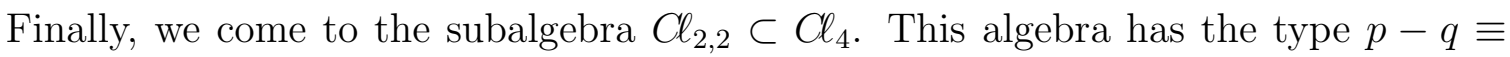
$0(\bmod 8)$ and $\mathbb{K} \simeq \mathbb{R}$. The mapping $C \ell_{2,2} \stackrel{\gamma}{\longrightarrow} \operatorname{End}_{\mathbb{R}}\left(\mathbb{S}_{4}\right)$ for the first primitive idempotent $f_{1}^{+}=\frac{1}{4}\left(1+\mathbf{e}_{13}\right)\left(1+\mathbf{e}_{24}\right)$ gives

$$
\begin{gathered}
\mathbb{K}=f_{1}^{+} C \ell_{2,2} f_{1}^{+} \simeq\{1\} \simeq \mathbb{R} . \\
\gamma_{1}=\left[\begin{array}{cccc}
0 & 1 & 0 & 0 \\
1 & 0 & 0 & 0 \\
0 & 0 & 0 & 1 \\
0 & 0 & 1 & 0
\end{array}\right], \quad \gamma_{2}=\left[\begin{array}{cccc}
0 & 0 & 1 & 0 \\
0 & 0 & 0 & -1 \\
1 & 0 & 0 & 0 \\
0 & 1 & 0 & 0
\end{array}\right], \\
\gamma_{3}=\left[\begin{array}{cccc}
0 & -1 & 0 & 0 \\
1 & 0 & 0 & 0 \\
0 & 0 & 0 & -1 \\
0 & 0 & 1 & 0
\end{array}\right], \quad \gamma_{4}=\left[\begin{array}{cccc}
0 & 0 & -1 & 0 \\
0 & 0 & 0 & 1 \\
1 & 0 & 0 & 0 \\
0 & -1 & 0 & 0
\end{array}\right] .
\end{gathered}
$$

As in the previous case of $C_{3,1}$ the spinor representation of the pseudoautomorphism $\mathcal{A} \rightarrow \overline{\mathcal{A}}$ is reduced to the unit matrix. For the idempotents $f_{1}^{ \pm}$we have

$$
\mathrm{PT}-\left(C \ell_{2,2}\right) \simeq\left\{\mathbf{1}_{4}, \gamma_{1234}, \gamma_{34}, \gamma_{12}\right\} \simeq \mathbb{Z}_{4}
$$




$$
C^{+,-,-} \simeq \mathbb{Z}_{4} \times \mathbb{Z}_{2} .
$$

It is easy to verify that all other primitive idempotents of $C_{2,2}$,

$$
\begin{gathered}
f_{2}^{ \pm}=\frac{1}{4}\left(1 \pm \mathbf{e}_{23}\right)\left(1 \pm \mathbf{e}_{14}\right), \quad f_{3}^{ \pm}=\frac{1}{4}\left(1 \pm \mathbf{e}_{14}\right)\left(1 \pm \mathbf{e}_{124}\right), \\
f_{4}^{ \pm}=\frac{1}{4}\left(1 \pm \mathbf{e}_{24}\right)\left(1 \pm \mathbf{e}_{1234}\right), \quad f_{5}^{ \pm}=\frac{1}{4}\left(1 \pm \mathbf{e}_{1}\right)\left(1 \pm \mathbf{e}_{23}\right), \\
f_{6}^{ \pm}=\frac{1}{4}\left(1 \pm \mathbf{e}_{2}\right)\left(1 \pm \mathbf{e}_{13}\right)
\end{gathered}
$$

generate spinor representations with $\mathrm{PT}_{-}\left(C_{2,2}\right) \simeq \mathbb{Z}_{4}$. Thus, the group $C^{+,-,-} \simeq$ $\mathbb{Z}_{4} \times \mathbb{Z}_{2}$ is an invariant fact for the algebra $C_{2,2}$.

\section{Acknowledgements}

I am grateful to Prof. R. Abłamowicz for helpful discussions about CLIFFORD.

\section{References}

[1] E. W. Mielke, Quantenfeldtheorie im de Sitter-Raum. Fortschr. Physik. 25 (1977) 401-457.

[2] T. S. Bunch, P.C.W. Davies, Quantum field theory in de Sitter space: Renormalization by point splitting. Proc. Roy. Soc. (London) A. 360 (1978) 117-134.

[3] B. Allen, Vacuum states in de Sitter space. Phys. Rev. D. 32 (1985) 3136-3164.

[4] A. D. Dolgov, M. B. Einhorn, V.I. Zakharov, The Vacuum of de Sitter Space. Acta Phys. Polon. B. 26 (1995) 65-90.

[5] J. Bros, U. Moschella, Two-point Functions and Quantum Fields in de Sitter Universe. Rev. Math. Phys. 8 (1996) 327-392.

[6] J. P. Gazeau, J. Renaud, M. V. Takook, Gupta-Bleuler quantization for minimally coupled scalar fields in de Sitter space. Class. Quant. Grav. 17 (2000) 1415-1434.

[7] P. Bartesaghi, J.P. Gazeau, U. Moschella, M. V. Takook, Dirac fields in the de Sitter model. Class. Quant. Grav. 18 (2001) 4373.

[8] R. F. Streater, A.S. Wightman, PCT, Spin and Statistics, and All That. W. A. Benjamin, Inc., New York-Amsterdam, 1964.

[9] W. Rarita, J. Schwinger, On a theory of particles with half-integral spin. Phys. Rev. 60 (1941) 61.

[10] V. Bargmann, E.P. Wigner, Group theoretical discussion of relativistic wave equations. Proc. Nat. Acad. USA. 34 (1948) 211-223. 
[11] I. M. Gel'fand, A.M. Yaglom, General relativistic-invariant equations and infinite-dimensional representations of the Lorentz group. Zh. Ehksp. Teor. Fiz. 18 (1948) 703-733.

[12] H. Joos, Zur darstellungstheorie der inhomogenen Lorentzgrouppe als grundlade quantenmechanische kinematick. Fortschr. Phys. 10 (1962) 65.

[13] S. Weinberg, Feinman rules for any spin I \& II \& III. Phys. Rev. B. 133 (1964) 1318-1332 \& 134 (1969) 882-896 \& 181 (1969) 1893-1899.

[14] T. D. Lee, G. C. Wick, Space inversion, time reversal, and other discrete symmetries. Phys. Rev. 148 (1966) 1385-1404.

[15] I. M. Gel'fand, R. A. Minlos, Z. Ya. Shapiro, Representations of the Rotation and Lorentz Groups and their Applications. Pergamon Press, Oxford, 1963.

[16] L. Michel, Invariance in quantum mechanics and group extension. In Group Theoretical Concepts and Methods in Elementary Particle Physics, Gordon \& Breach, New York, 1964, pp. 135-200.

[17] T. K. Kuo, Internal-symmetry groups and their automorphisms. Phys. Rev. D4 (1971) 3620-3637.

[18] Z. K. Silagadze, On the internal parity of antiparticles. Sov. J. Nucl. Phys. 55 (1992) 392-396.

[19] I. L. Buchbinder, D. M. Gitman, A. L. Shelepin, Discrete symmetries as automorphisms of proper Poincaré group. Int. J. Theor. Phys. 41 (2002) 753-790.

[20] D. M. Gitman, A. L. Shelepin, Fields on the Poincaré Group: Arbitrary Spin Description and Relativistic Wave Equations. Int. J. Theor. Phys. 40 (2001) 603-684.

[21] V. V. Varlamov, Discrete Symmetries and Clifford Algebras. Int. J. Theor. Phys. 40 (2001) 769-805; arXiv:math-ph/0009026 (2000).

[22] V. V. Varlamov, Group Theoretical Interpretation of CPT-theorem. In C. V. Benton, ed., Mathematical Physics Research at the Cutting Edge, Nova Science Publishers, New York, 2004, pp. 51-100; arXiv:math-ph/0306034 (2003).

[23] P. Budinich, A. Trautman, The Spinorial Chessboard. Springer, Berlin, 1988.

[24] R. Penrose, W. Rindler, Spinors and space-time. Vol. 2. Spinor and twistor methods in space-time geometry. Cambridge Monographs on Mathematical Physics, 2nd edn. Cambridge University Press, Cambridge and New York, 1988.

[25] J. A. Schouten, On the geometry of spin spaces. Indag. Math. 11 (1949) 3,4,5.

[26] P. K. Rashevskii, The Theory of Spinors. Uspekhi Mat. Nauk. 10 (1955) 3-110; English translation in Amer. Math. Soc. Transl. (Ser. 2). 6 (1957) 1. 
[27] P. K. Rashevskii, About Mathematical Foundations of Quantum Electrodynamics. (in Russian) Uspekhi Mat. Nauk 13 (1958) 3-110.

[28] V. V. Varlamov, Universal Coverings of Orthogonal Groups. Adv. Appl. Clifford Algebras. 14 (2004) 81-168; arXiv:math-ph/0405040 (2004).

[29] M. Socolovsky, The CPT group of the Dirac field. Int. J. Theor. Phys. 43 (2004) 1941-1967; arXiv:math-ph/0404038 (2004).

[30] V. V. Varlamov, The CPT Group in the de Sitter Space. Annales de la Fondation Louis de Broglie. 29 (2004) 969-987; arXiv:math-ph/0406060 (2004).

[31] V.V. Varlamov, CPT groups for spinor field in de Sitter space. Phys. Lett. B. 631 (2005) 187-191; arXiv:math-ph/0508050 (2005).

[32] V.V. Varlamov, CPT Groups of Higher Spin Fields. Int. J. Theor. Phys. 51 (2012) 1453-1481; arXiv: 1107.4156 [math-ph] (2011).

[33] N. Salingaros, Realization, extension, and classification of certain physically important groups and algebras. J. Math. Phys. 22 (1981) 226-232.

[34] N. Salingaros, On the classification of Clifford algebras and their relation to spinors in $n$ dimensions. J. Math. Phys. 23 (1982) 1-7.

[35] N. Salingaros, The relationship between finite groups and Clifford algebras. J. Math. Phys. 25 (1984) 738-742.

[36] H.W. Braden, $N$-dimensional spinors: Their properties in terms of finite groups. J. Math. Phys. 26 (1985) 613-620.

[37] R. Shaw, Finite geometry, Dirac groups and the table of real Clifford algebras. Univ. of Hull Maths Research Report. 7 (1994) 1.

[38] Yu. M. Shirokov, Group theoretical analysis of the foundations of relativistic quantum mechanics. IV, V. Zh. Ehksp. Teor. Fiz. 34 (1958) 717-724; 36 (1959) 879-888.

[39] Yu. M. Shirokov, Spacial and time reflections in the relativistic theory. Zh. Ehksp. Teor. Fiz. 38 (1960) 140-150.

[40] L. Dąbrowski, Group Actions on Spinors. Bibliopolis, Naples, 1988.

[41] A. Chamblin, On the Obstructions to Non-Cliffordian Pin Structures. Commun. Math. Phys. 164 (1994) 67-87.

[42] P. A. M. Dirac, The electron wave equation in de Sitter space. Annals of Math. 36 (1935) 657.

[43] R. Brauer, H. Weyl, Spinors in n dimensions. Amer. J. Math. 57 (1935) 425-449. 
[44] P. Lounesto, Clifford Algebras and Spinors. Cambridge Univ. Press, Cambridge, 2001.

[45] J. Radon, Lineare Scharen orthogonaler Matrizen. Abh. Math. Seminar Hamburg. 1 (1922) 1-24.

[46] A. Hurwitz, Uber die Komposition der quadratischen Formen. Math. Ann. 88 (1923) $1-25$.

[47] R. Abłamowicz, B. Fauser, On the transposition anti-involution in real Clifford algebras I: The transposition map. Linear and Multilinear Algebra 59(12) (2011) 1331-1358; arXiv: 1005.3554 [math-ph] (2010).

[48] R. Abłamowicz, B. Fauser, On the transposition anti-involution in real Clifford algebras II: Stabilizer groups of primitive idempotents. Linear and Multilinear Algebra 59(12) (2011) 1359-1381; arXiv: 1005.3558 [math-ph] (2010).

[49] R. Abłamowicz, B. Fauser, On the Transposition Anti-involution in Real Clifford Algebras III: The Automorphism Group of the Transposition Scalar Product on Spinor Spaces. arXiv: 1102.3304 [math-ph] (2011).

[50] R. Abłamowicz, Spinor Representations of Clifford Algebras: A Symbolic Approach. CPC Thematic Issue 'Computer Algebra in Physics Research', Physics Communications 115 (1998) 510-535.

[51] S. S. Schweber, An Introduction to Relativistic Quantum Field Theory. Harper and Row, New York, 1961.

[52] A. Z. Capri, Relativistic Quantum Mechanics and Introduction to Quantum Field Theory. World Scientific, New Jersey, 2002.

[53] E. P. Wigner, Unitary Representations of the Inhomogeneous Lorentz Group Including Reflections. in F. Gürsey, ed., Group Theoretical Concepts and Methods in Elementary Particle Physics, Gordon \& Breach, New York, 1964.

[54] D. V. Ahluwalia, M. B. Johnson, T. Goldman, A Bargmann-Wightman-Wigner Type Quantum Field Theory. Phys. Lett. B 316(1) (1993) 102-108.

[55] D. V. Ahluwalia, A New Type of Massive Spin-One Boson: and its Relation with Maxwell Equations. The Present Status of the Quantum Theory of Light, S. Jeffers et al (eds.) pp. 443-457 (1997). arXiv: hep-th/9509116 (1995).

[56] V. V. Dvoeglazov, P, C and T for Truly Neutral Particles. AIP Conf. Proc. 1232 (2010) 235-242; arXiv: 0909.4743 [hep-th] (2009).

[57] V.V. Dvoeglazov, How to construct self/anti-self charge conjugate states for higher spins?. AIP Conf. Proc. 1488 (2012) 248-256; arXiv: 1210.4401 [hep-th] (2012). 
[58] R. da Rocha, W. A. Rodrigues Jr., Where are ELKO Spinor Fields in Lounesto Spinor Field Classification?. Mod. Phys. Lett. A 21 (2006) 65-74; arXiv: math-ph/0506075 (2005).

[59] S. Moradi, S. Rouhani, M. V. Takook, Discrete Symmetries for Spinor Field in de Sitter Space. Phys. Lett. B. 613 (2005) 74-82.

[60] V.V. Varlamov, Cyclic structures of Cliffordian supergroups and particle representations of Spin $_{+}(1,3)$. arXiv: 1207.6162 [math-ph] (2012), to appear in Adv. Appl. Clifford Algebras.

[61] J.S. R. Chisholm, R. S. Farwell, Properties of Clifford Algebras for Fundamental Particles. in W. Baylis, ed., Clifford (Geometric) Algebras, Birkhäuser, Boston, 1996, pp. 365-388.

[62] P. A. M. Dirac, The principles of quantum mechanics. Clarendon Press, Oxford, 1958. 